\title{
ON THE CONDITIONAL EXPECTATION AND CONVERGENCE PROPERTIES OF RANDOM SETS
}

\author{
NIKOLAOS S. PAPAGEORGIOU
}

\begin{abstract}
In this paper we study random sets, with values in a separable Banach space. First we establish several useful properties of the set-valued conditional expectation and then prove some convergence theorems for set-valued amarts and uniform amarts, using the weak, Kuratowski-Mosco and Hausdorff modes of set convergence.
\end{abstract}

\section{INTRODUCTION}

Since the early seventies, there has been an extensive development of the theory of set-valued random processes. The motivation came from both the theory and the applications. Set-valued random processes are the natural generalization of random processes and it is interesting to know whether we can develop for them a convergence and representation theory analogous to the one existing for point-valued processes. This line of theoretical research can be traced in the works of Alo-de Korvin [1], Bagchi [5], Costé [8], Dam [9], Daures [10], Hiai-Umegaki [17], Hiai [18], Luu [25], Neveu [27], Papageorgiou [29, 30, 34, 35] and Wang-Xue [45]. On the other hand, the works of de Korvin-Kleyle [23], Papageorgiou [32, 33], Salinetti-Wets [40, 41], and Yovits-Foulk-Rose [47] illustrate the importance of set-valued random processes in various applied fields, like optimization (see [40, 41]), mathematical economics (see $[32,33]$ ) and in the analysis of uncertain information systems (see [23, 47]).

The purpose of this paper is to establish some new properties of the setvalued conditional expectation and prove some convergence theorems for setvalued amarts and uniform amarts. Given that these two classes incorporate setvalued martingales and quasi-martingales (see section 5), we can view our work here as the continuation of the recent important work of Wang-Xue [45], who obtained the most general convergence theorems for set-valued semimartingales, extending among other things some of the results of Papageorgiou [34].

\section{Preliminaries}

Throughout this paper $(\Omega, \Sigma, \mu)$ will be a complete probability space, $\Sigma_{0}$ a sub- $\sigma$-field of $\Sigma,\left\{\Sigma_{n}\right\}_{n \geq 1}$ an increasing sequence of sub- $\sigma$-fields of $\Sigma$ such

Received by the editors December 23, 1993 and, in revised form, July 9, 1994.

1991 Mathematics Subject Classification. Primary 60B12, 60G42, 60G48.

Key words and phrases. Set-valued conditional expectation, set-valued amarts and uniform amarts, weak convergence, Kuratowski-Mosco convergence, Hausdorff metric, optional sampling. 
that $\Sigma=\sigma\left(\bigcup_{n>1} \Sigma_{n}\right)$ and $X$ a separable Banach space. We will be using the following notations:

$$
P_{f(c)}(X)=\{A \subseteq X: \text { nonempty, closed (and convex) }\}
$$

and

$$
P_{(w) k(c)}(X)=\{A \subseteq X \text { : nonempty, (weakly) compact (and convex) }\} .
$$

Also if $A \in 2^{X} \backslash\{\varnothing\}$, we denote by $|A|$ the "norm" of the set $A$, i.e. $|A|=$ $\sup \{\|x\|: x \in A\}$; by $\sigma(\cdot, A)$ the support function of $A$, i.e. $\sigma\left(x^{*}, A\right)=$ $\sup \left[\left(x^{*}, x\right): x \in A\right]$ for all $x^{*} \in X^{*} ;$ and finally by $d(\cdot, A)$ the distance function from the set $A$, i.e. $d(z, A)=\inf [\|z-x\|: x \in A]$, for every $z \in X$.

A multifunction (set-valued function, random set) $F: \Omega \rightarrow P_{f}(X)$ is said to be measurable, if for all $z \in X$ the $\mathbb{R}_{+}$-valued function $\omega \rightarrow d(z, F(\omega))$ is measurable. In fact since we have assumed $\Sigma$ to be $\mu$-complete, this definition of measurability of $F(\cdot)$ is equivalent to saying that

$$
\operatorname{Gr} F=\{(\omega, x) \in \Omega \times X: x \in F(\omega)\} \in \Sigma \times B(X)
$$

with $B(X)$ being the Borel $\sigma$-field of $X$ (graph measurability). In general, graph measurability is the weaker notion. Further details on the measurability of multifunctions, with a detailed historical survey of the development of the theory, can be found in the paper of Wagner [44]. One historical remark is appropriate here. As was pointed out by Wang-Xue [45] and we concur, one of the first to consider the problem of measurability of set-valued functions was Robbins [37, 38]. Unfortunately his work was subsequently overlooked by the people working in this area.

By $S_{F}^{1}$ we will denote the set of selectors of $F(\cdot)$, that belong in the LebesgueBochner space $L^{1}(\Omega, X)$; i.e. $S_{F}^{1}=\left\{f \in L^{1}(\Omega, X): f(\omega) \in F(\omega) \mu\right.$-a.e. $\}$. It may happen that this set is empty. A straightforward application of Aumann's selection theorem (cf. Wagner [44, Theorem 5.10]) reveals that, for a graph measurable multifunction $F: \Omega \rightarrow 2^{X} \backslash\{\varnothing\}$, the set $S_{F}^{1}$ is nonempty if and only if there exists $\varphi(\cdot) \in L^{1}(\Omega)$ s.t. $\inf \{\|x\|: x \in F(\omega)\} \leq \varphi(\omega) \mu$-a.e. We say that $F: \Omega \rightarrow P_{f}(X)$ is integrably bounded if $F(\cdot)$ is measurable and $\omega \rightarrow$ $|F(\omega)| \in L^{1}(\Omega)$. So for an integrably bounded multifunction, we see that $S_{F}^{1} \neq$ $\varnothing$. Using the set $S_{F}^{1}$, we can define a set-valued integral for $F(\cdot)$, by setting $\int_{\Omega} F(\omega) d \mu(\omega)=\left\{\int_{\Omega} f(\omega) d \mu(\omega): f \in S_{F}^{1}\right\}$. The vector-valued integrals in this definition are understood in the sense of Bochner.

Let $F: \Omega \rightarrow P_{f}(X)$ be a measurable multifunction with $S_{F}^{1} \neq \varnothing$. Following Hiai-Umegaki [17], we define the set-valued conditional expectation of $F(\cdot)$ with respect to $\Sigma_{0}$ to be the $\Sigma_{0}$-measurable multifunction $E^{\Sigma_{0}} F: \Omega \rightarrow P_{f}(X)$ for which we have that $S_{E^{\Sigma_{0}}}^{1}\left(\Sigma_{0}\right)=\operatorname{cl}\left\{E^{\Sigma_{0}} f: f \in S_{F}^{1}\right\}$, the closure taken in $L^{1}(\Omega, X)$. Note that the set $K=\left\{E^{\Sigma_{0}} f: f \in S_{F}^{1}\right\}$ is $\Sigma_{0}$-decomposable; i.e. if $\left(g_{1}, g_{2}, A\right) \in K \times K \times \Sigma_{0}$, then $\chi_{A} g_{1}+\chi_{A^{c}} g_{2} \in K$. Hence $\operatorname{cl} K$ is $\Sigma_{0^{-}}$ decomposable and so by Theorem 3.1 of Hiai-Umegaki [17], there exists a unique (up to $\mu$-null sets) $\Sigma_{0}$-measurable multifunction $E^{\Sigma_{0}} F: \Omega \rightarrow P_{f}(X)$ for which $S_{E^{\Sigma_{0} F}}^{1}\left(\Sigma_{0}\right)=\mathrm{cl} K$. So the conditional expectation $E^{\Sigma_{0}} F(\cdot)$ of $F(\cdot)$ with respect to $\Sigma_{0}$ is well-defined. If $F(\cdot)$ is convex-valued (integrably bounded), then so is $E^{\Sigma_{0}} F(\cdot)$ (cf. Hiai-Umegaki [17]). The set-valued conditional expectation behaves much like the usual point-valued conditional expectation. So 
in particular, if $F(\cdot)$ is $\Sigma_{0}$-measurable, then $E^{\Sigma_{0}} F(\omega)=F(\omega) \mu$-a.e.; if $\Sigma_{0}^{\prime}$, $\Sigma_{0}$ are sub- $\sigma$-fields of $\Sigma$ and $\Sigma_{0}^{\prime} \subseteq \Sigma_{0}$, then $E^{\Sigma_{0}^{\prime}}\left(E^{\Sigma_{0}} F\right)=E^{\Sigma_{0}^{\prime}} F \quad \mu$-a.e. and finally, for every $A \in \Sigma_{0}$,

$$
\operatorname{cl} \int_{A}^{\left(\Sigma_{0}\right)} E^{\Sigma_{0}} F(\omega) d \mu(\omega)=\operatorname{cl} \int_{A} F(\omega) d \mu(\omega)
$$

where

$$
\int_{A}^{\left(\Sigma_{0}\right)} E^{\Sigma_{0}} F(\omega) d \mu(\omega)=\left\{\int_{A} f(\omega) d \mu(\omega): f \in L^{1}\left(\Sigma_{0}, X\right), f(\omega) \in F(\omega) \mu \text {-a.e. }\right\} \text {. }
$$

In addition if $F(\cdot)$ is $P_{f c}(X)$-valued, then

$$
\operatorname{cl} \int_{A} E^{\Sigma_{0}} F(\omega) d \mu(\omega)=\operatorname{cl} \int_{A} F(\omega) d \mu(\omega)
$$

(cf. Hiai-Umegaki [17]).

A sequence of multifunctions $F_{n}: \Omega \rightarrow P_{f}(X)$ is said to be adapted to $\Sigma_{n}$ if, for every $n \geq 1, F_{n}(\cdot)$ is $\Sigma_{n}$-measurable. An adapted sequence $\left\{F_{n}, \Sigma_{n}\right\}_{n \geq 1}$ is said to be a set-valued martingale if and only if, for every $n \geq 1, E^{\Sigma_{n}} F_{n+1}(\omega)=$ $F_{n}(\omega) \mu$-a.e. An adapted sequence $\left\{F_{n}, \Sigma_{n}\right\}_{n \geq 1}$ is said to be a set-valued quasimartingale if and only if $\sum_{n \geq 1} \Delta\left(F_{n}, E^{\Sigma_{n}} F_{n+1}\right)<\infty$, where $\Delta\left(F_{n}, E^{\Sigma_{n}} F_{n+1}\right)=$ $\int_{\Omega} h\left(F_{n}(\omega), E^{\Sigma_{n}} F_{n+1}(\omega)\right) d \mu(\omega)$ and $h(\cdot, \cdot)$ denotes the usual Hausdorff generalized metric on $P_{f}(X)$. It is obvious that a set-valued martingale is a setvalued quasi-martingale, but the converse is not true in general. A function $\tau: \Omega \rightarrow N_{+} \cup\{+\infty\}$ is said to be a stopping-time with respect to $\left\{\Sigma_{n}\right\}_{n \geq 1}$ if, for each $n \geq 1,\{\tau=n\}=\{\omega \in \Omega: \tau(\omega)=n\} \in \Sigma_{n}$. The set of all stopping times is denoted by $T^{*}$. We can partially order $T^{*}$ in the obvious way; namely, if $\tau_{1}, \tau_{2} \in T^{*}$, we say that $\tau_{1} \leq \tau_{2}$ if and only if $\tau_{1}(\omega) \leq \tau_{2}(\omega)$ for all $\omega \in \Omega$. By $T$ we will denote the subset of $T^{*}$ consisting of all bounded stopping times. So $\tau \in T$ if and only if $\tau \in T^{*}$ and the range of $\tau(\cdot)$ is a finite set in $\mathbb{N}_{+}$. The order induced on $T$ by $T^{*}$ has the property that $\mathbb{N}_{+}$is cofinal in $T$. Given $\tau \in T$, we define

$$
\Sigma_{\tau}=\left\{A \in \Sigma: A \cap\{\tau=n\} \in \Sigma_{n}, n \geq 1\right\}
$$

Then $\left\{\Sigma_{\tau}\right\}_{\tau \in T}$ is an increasing family of sub- $\sigma$-fields of $\Sigma$. Also we define $F_{\tau}(\omega)=F_{\tau(\omega)}(\omega)$ for all $\omega \in \Omega$. From Luu [25] we know that $F_{\tau}: \Omega \rightarrow P_{f}(X)$ is $\Sigma_{\tau}$-measurable. In section 4 , we show that if $\left\{F_{n}, \Sigma_{n}\right\}_{n \geq 1}$ is a set-valued martingale, then so is the net $\left\{F_{\tau}, \Sigma_{\tau}\right\}_{\tau \in T}$ ("optional sampling theorem"). Now let $\left\{F_{n}, \Sigma_{n}\right\}_{n \geq 1}$ be an adapted set-valued random process. In analogy with the point-valued case (cf. Egghe [15]), we say that $\left\{F_{n}, \Sigma_{n}\right\}_{n \geq 1}$ is a set-valued amart, if the net $\left\{\mathrm{cl} \int_{\Omega} F_{\tau}\right\}_{\tau \in T}$ is $h$-convergent (i.e. convergent for the Hausdorff generalized metric on $\left.P_{f}(X)\right)$. Since $\left(P_{f}(X), h\right)$ is a complete generalized metric space, there is some $K \in P_{f}(X)$ such that $h\left(\operatorname{cl} \int_{\Omega} F_{\tau} d \mu, K\right) \rightarrow 0$ for $\tau \in T$. We will say that $\left\{F_{n}, \Sigma_{n}\right\}_{n \geq 1}$ is a set-valued uniform amart if and only if $\lim _{\tau \in T} \sup _{\sigma \geq \tau} \Delta\left(F_{\tau}, E^{\Sigma_{\tau}} F_{\sigma}\right)=0$ (recall that $\Delta\left(F_{\tau}, E^{\Sigma_{\tau}} F_{\sigma}\right.$ ) = $\left.\int_{\Omega} h\left(F_{\tau}, E^{\Sigma_{\tau}} F_{\sigma}\right) d \mu(\omega)\right)$. This definition generalizes in a natural way to setvalued random processes, the concept of a point-valued uniform amart (cf. Bellow [6]). Clearly a set-valued uniform amart is a set-valued amart.

In what follows by $\mathscr{L}_{f}^{1}(\Sigma, X)$ we will denote the set of all equivalence classes of integrably bounded multifunctions $F: \Omega \rightarrow P_{f}(X)$ where two multifunctions 
$F_{1}, F_{2}$ are considered to be identical if and only if $F_{1}(\omega)=F_{2}(\omega) \mu$-a.e. Furnished with the metric $\Delta(F, G)=\int_{\Omega} h(F(\omega), G(\omega)) d \mu(\omega), \mathscr{L}_{f}^{1}(\Sigma, X)$ becomes a complete metric space. Similarly, we can define $\mathscr{L}_{f c}^{1}(\Sigma, X)$ and $\mathscr{L}_{w k c}^{1}(\Sigma, X)$. Note that $\mathscr{L}_{f c}^{1}(X)$ is a closed subspace of the metric space $\left(\mathscr{L}_{f}^{1}(\Sigma, X), \Delta\right)$; hence $\left(\mathscr{L}_{f c}^{1}(X), \Delta\right)$ is itself a complete metric space.

An operator $L: \Sigma \times L^{p}(\Omega, X) \rightarrow \overline{\mathbb{R}}=\mathbb{R} \cup\{+\infty\}, 1 \leq p \leq \infty$, will be said to be local on $\Sigma$ if and only if, for every $u, v \in L^{p}(\Omega, X)$ and every $A \in \Sigma, \chi_{A} u=\chi_{A} v \quad \mu$-a.e. implies that $L(A, u)=L(A, v)$. We will say that $L$ is additive on $\Sigma$ if, for every $u \in L^{p}(\Omega, X)$ and for every $A_{1}, A_{2} \in \Sigma$, $A_{1} \cap A_{2}=\varnothing$ implies that $L\left(A_{1} \cup A_{2}, u\right)=L\left(A_{1}, u\right)+L\left(A_{2}, u\right)$. Finally we will say that $L(\cdot, \cdot)$ is proper if there exists $u_{0} \in L^{p}(\Omega, X)$ such that $L\left(A, u_{0}\right)<\infty$ for all $A \in \Sigma$.

Recall that if $Y$ is a Banach space and $f: \Omega \rightarrow Y$, we say that $f(\cdot)$ is scalarly integrable if, for all $x^{*} \in X^{*},\left(x^{*}, f(\cdot)\right) \in L^{1}(\Omega)$. If for every $A \in \Sigma$, there exists $x_{A} \in X$ such that $\left(x^{*}, x_{A}\right)=\int_{A}\left(x^{*}, f(\omega)\right) d \mu(\omega)$, then we say that $F(\cdot)$ is Pettis-integrable and we write $x_{A}=P-\int_{A} f(\omega) d \mu(\omega)$. Clearly every Bochner integrable function is Pettis integrable, but the converse is not in general true. A Banach space $X$ is said to have the Radon-Nikodym Property (RNP) (resp. the Weak Radon-Nikodym Property (WRNP)) if, for every complete probability space $(\Omega, \Sigma, \mu)$ and for every vector measure $m: \Sigma \rightarrow X$ of bounded variation such that $m \ll \mu$, there exists a Bochner integrable function (resp. a Pettisintegrable function) $f(\cdot)$ such that for every $A \in \Sigma$

$$
m(A)=\int_{A} f(\omega) d \mu(\omega) \quad\left(\text { resp. } m(A)=P-\int_{A} f(\omega) d \mu(\omega)\right) .
$$

It is an immediate consequence of the Pettis measurability theorem (see Diestel-Uhl [11, Theorem 2, p. 42]) that on separable Banach spaces RNP and WRNP are equivalent. In general, WRNP is of course weaker than RNP.

Let $\left\{A_{n}\right\}_{n \geq 1} \subseteq 2^{X} \backslash\{\varnothing\}$. We define

$s-\underline{\lim } A_{n}=\left\{x \in X: \lim d\left(x, A_{n}\right)=0\right\}=\left\{x \in X: x=\lim x_{n}, x_{n} \in A_{n}, n \geq 1\right\}$ and

$$
w-\varlimsup \lim A_{n}=\left\{x \in X: x=w-\lim x_{n_{k}}, x_{n_{k}} \in A_{n_{k}}, n_{1}<n_{2}<\cdots<n_{k}<\cdots\right\}
$$

(here $s$-denotes the strong topology on $X$ and $w$ - the weak topology). Note that we always have $s-\underline{\lim } A_{n} \subseteq w-\overline{\lim } A_{n}$. We say that the $A_{n}$ 's converge to $A$ in the Kuratowski-Mosco sense, denoted by $A_{n} \stackrel{\text { K-M }}{\rightarrow} A$ if and only if $w-\varlimsup \lim A_{n}=A=s-\underline{\lim } A_{n}$. Also we will say that the $A_{n}$ 's converge to $A$ weakly (or scalarly), denoted by $A_{n} \stackrel{\text { w }}{\rightarrow} A$ if and only if, for all $x^{*} \in X^{*}$, $\sigma\left(x^{*}, A_{n}\right) \rightarrow \sigma\left(x^{*}, A\right)$. The notions of $\mathrm{K}-\mathrm{M}$ and weak convergence of sets are in general disjoint and are both implied by convergence in the Hausdorff generalized pseudometric (resp. metric) on $2^{X}$ (resp. on $P_{f}(X)$ ). Also if $\operatorname{dim} X<\infty$ and $\left\{A_{n}, A\right\}_{n \geq 1} \subseteq P_{k}(X)$, then all three types of convergence coincide (cf. Attouch [3] and Klein-Thompson [22]). Recall that if $A, C \in$ $2^{X} \backslash\{\varnothing\}$, the Hausdorff distance of $A$ and $C$ is defined by

$$
h(A, C)=\max \left[\sup _{a \in A} d(a, C), \sup _{c \in C} d(c, A)\right] .
$$


Furthermore, if $A, C \in P_{f c}(X)$ and are bounded, then

$$
h(A, C)=\sup \left[\left|\sigma\left(x^{*}, A\right)-\sigma\left(x^{*}, C\right)\right|:\left\|x^{*}\right\| \leq 1\right]
$$

(cf. Hörmander [19]).

Finally a topological $(V, \tau)$ is a Polish space, if $\tau$ is metrizable by some metric $d$ and $(V, d)$ is a complete separable metric space. A Souslin space is a Hausdorff topological space, which is the continuous image of a Polish space. A Polish space is a Souslin space and so is a separable Banach space furnished with the weak topology. So a Souslin space is always separable, but need not be metrizable. More generally, if $X$ is a separable Banach space, then $X_{w^{*}}^{*}$ (the dual space $X^{*}$ equipped with the $w^{*}$-topology) is a Souslin space. Two comparable Souslin topologies define the same Borel subsets.

\section{THE CONDITIONAL EXPECTATION OF A RANDOM SET}

In this section we establish some useful properties of the set-valued conditional expectation. In doing this we also obtain some peripheral results which are actually of independent interest. So we see how the structure of $S_{F}^{1}$ determines the pointwise properties of $F(\cdot)$, we prove a representation theorem for nonlinear, local additive operators on $L^{\infty}\left(\Omega, X_{w^{*}}^{*}\right)$, from which we derive an expression for the set-valued conditional expectation $E^{\Sigma_{0}} F(\cdot)$ and finally we characterize the elements in $S_{E^{\Sigma_{0} F}}^{1}$.

As we already mentioned in section $2,(\Omega, \Sigma, \mu)$ is a complete probability space and $X$ a separable Banach space. Additional hypotheses will be introduced as needed. We start with a result which can be viewed as a converse to Proposition 3.1 of Papageorgiou [29]. Recall that according to that proposition, if $F: \Omega \rightarrow P_{w k c}(X)$ is integrably bounded, then $S_{F}^{1}$ is $w$-compact and convex in $L^{1}(\Omega, X)$.

Proposition 1. If $X$ is weakly sequentially complete, $X^{*}$ has the WRNP and $F: \Omega \rightarrow 2^{X} \backslash\{\varnothing\}$ is a graph measurable multifunction such that $S_{F}^{1}$ is nonempty, bounded closed and convex,

then $F(\cdot)$ is $\mu$-a.e. $P_{w k c}(X)$-valued and integrably bounded.

Proof. From Hiai-Umegaki [17, Theorems 3.1 and 3.2], we know that $F: \Omega \rightarrow$ $P_{f c}(X)$ and is integrably bounded. Hence for all $\omega \in \Omega \backslash N, \mu(N)=0, F(\omega)$ is bounded. We will show that in fact $F(\omega) \in P_{w k c}(X)$. Suppose that for some $\omega \in \Omega \backslash N, F(\omega)$ is not $w$-compact. From the Eberlein-Smulian theorem, we know that there exists a sequence $\left\{x_{n}\right\}_{n \geq 1}$ in $F(\omega)$ with no weakly convergent subsequence. Since $\left\{x_{n}\right\}_{n \geq 1}$ is bounded and $X$ is weakly sequentially complete, from Rosenthal's dichotomy theorem [39], we have that $\left\{x_{n}\right\}_{n \geq 1}$ is an $l^{1}$-sequence. So $l^{1}$ embeds into $X$, a contradiction to the fact that $X^{*}$ has the WRNP (cf. Musial [26]). Q.E.D.

Remarks. (i) If $X^{*}$ has the RNP, then the result is immediate because, in this case, $X$ is reflexive (cf. Diestel-Uhl [11, Corollary 11, p. 198]).

(ii) Our result partially extends Theorem 3.6 (i) of Klei [21].

In fact, we can have the following more general result. Recall that a subset $K \subseteq L^{1}(\Omega, X)$ is decomposable if, for all $\left(f_{1}, f_{2}, A\right) \in L^{1}(\Omega, X) \times L^{1}(\Omega, X) \times$ $\Sigma, \chi_{A} f_{1}+\chi_{A^{c}} f_{2} \in K$. 
Proposition 2. If $X$ is weakly sequentially complete, $X^{*}$ has the WRNP and $K \subseteq L^{1}(\Omega, X)$ is decomposable and bounded,

then $K$ is reiatively weakly compact in $L^{1}(\Omega, X)$.

Proof. We claim that $K$ is uniformly integrable. To this end let $|K|=\{\|f(\cdot)\|$ : $f \in K\}$ and let $h=$ esssup $|K|$ (cf. Neveu [28]). We need to show that $h(\cdot) \in$ $L^{1}(\Omega)$. From Proposition VI-1-1, p. 121 of Neveu [28], we know that $h(\omega)=$ $\sup _{n \geq 1}\left\|f_{n}(\omega)\right\| \quad \mu$-a.e. Furthermore, the decomposability of $K$ implies that $|K|$ is directed upwards. Then from the above-mentioned result of Neveu, we know that we can have $\left\|f_{n}(\omega)\right\| \uparrow h(\omega) \quad \mu$-a.e. Since $K$ is bounded, an application of the monotone convergence theorem tells us that $h \in L^{1}(\Omega)$. Hence $K$ is uniformly integrable. Also because $X^{*}$ has the WRNP, we have that $l^{1}$ does not embed in $X$. So by Corollary 9 of Bourgain [7] (see also Pisier [36]), $K$ does not contain a sequence equivalent to the standard $l^{1}$-basis. So if $\left\{u_{n}\right\}_{n \geq 1}$ is a sequence in $K$, by Rosenthal's dichotomy theorem [39], we have $\left\{u_{n}\right\}_{n \geq 1}$ has a weakly Cauchy subsequence. Finally since $X$ is weakly complete, from Talagrand [43], we know that $L^{1}(\Omega, X)$ is weakly sequentially complete and so we conclude that $\bar{K}^{w}$ is weakly compact. Q.E.D.

Now we turn our attention to the set-valued conditional expectation.

Proposition 3. If $F: \Omega \rightarrow P_{w k c}(X)$ is an integrably bounded multifunction,

$$
\text { then } E^{\Sigma_{0}} F(\omega) \in P_{w k c}(X) \mu \text {-a.e. }
$$

Proof. From Proposition 3.1 of Papageorgiou [29] (see also Theorem 3.6 (ii) of Klei [21]), we know that $S_{F}^{1}$ is $w$-compact in $L^{1}(\Omega, X)$. So $E^{\Sigma_{0}} S_{F}^{1}$ is $w$ compact and convex in $L^{1}\left(\Sigma_{0}, X\right)$. But recall that by definition $S_{E \Sigma_{0}}^{1}\left(\Sigma_{0}\right)=$ $\operatorname{cl}\left\{E^{\Sigma_{0}} S_{F}^{1}\right\}$. Thus invoking Corollary 1.6 of Hiai-Umegaki [17] and Theorem 3.6 (i) of Klei [21], we conclude that $E^{\Sigma_{0}} F(\omega) \in P_{w k c}(X) \mu$-a.e. Q.E.D.

In fact using Proposition 2, we can have the following alternative version of the above result.

Proposition 4. If $X$ is weakly sequentially complete, $X^{*}$ has the WRNP and $F: \Omega \rightarrow P_{f}(X)$ is integrably bounded,

then, for $\mu$-almost all $\omega \in \Omega,{\overline{E^{\Sigma_{0}} F(\omega)}}^{w}$ is $w$-compact.

Proof. Note that $S_{E^{\Sigma_{0} F}}^{1}\left(\Sigma_{0}\right)$ is a decomposable bounded subset of $L^{1}\left(\Sigma_{0}, X\right)$. Then according to Proposition $2, S_{E^{\Sigma_{0} F}}^{1}\left(\Sigma_{0}\right)$ is relatively weakly compact. To conclude the proof, apply Theorem 3.6 (i) of Klei [21]. Q.E.D.

A set $A \in \Sigma$ is said to be a $\Sigma_{0}$-atom if and only if for all $A^{\prime} \in \Sigma, A^{\prime} \subseteq A$, there exists $B \in \Sigma_{0}$ such that $\mu\left(A^{\prime} \Delta(A \cap B)\right)=0$ or equivalently $\chi_{A^{\prime}}(\bar{\omega})=$ $\chi_{A \cap B}(\omega) \mu$-a.e. (cf. Hanen-Neveu [16]).

Proposition 5. If $\Sigma$ has no $\Sigma_{0}$-atoms, $X$ is weakly sequentially complete, $X^{*}$ has the WRNP $\overline{\text { and }} F: \Omega \rightarrow P_{f}(X)$ is integrably bounded,

$$
\text { then } E^{\Sigma_{0}} F(\omega) \in P_{w k c}(X) \mu \text {-a.e. }
$$

Proof. Recall that $E^{\Sigma_{0}} F(\cdot)$ is $P_{f}(X)$-valued. Also since $\Sigma$ has no $\Sigma_{0}$-atoms, from Dynkin-Evstigneev [13], we know that $E^{\Sigma_{0}} F(\omega)$ is $\mu$-a.e. convex. So for 
$\mu$-almost all $\omega \in \Omega, E^{\Sigma_{0}} F(\omega)$ is closed, convex, hence weakly closed. Applying Proposition 4 we conclude that $E^{\Sigma_{0}} F(\omega) \in P_{w k c}(X) \mu$-a.e. Q.E.D.

Now we turn our attention to the support function of the set-valued conditional expectation. Let $F: \Omega \rightarrow P_{f c}(X)$ be a measurable multifunction with $S_{F}^{1} \neq \varnothing$. The function $\left(\omega, x^{*}\right) \rightarrow \sigma\left(x^{*}, F(\omega)\right)$ is jointly measurable and $w^{*}$-l.s.c. and sublinear in $x^{*} \in X^{*}$. By the conditional expectation of $\left(\omega, x^{*}\right) \rightarrow \sigma\left(x^{*}, F(\omega)\right)$ with respect to the sub- $\sigma$-field $\Sigma_{0}$, we mean a function $\varphi: \Omega \times X^{*} \rightarrow \overline{\mathbb{R}}=\mathbb{R} \cup\{+\infty\}$, which is $\Sigma_{0} \times B\left(X_{w^{*}}^{*}\right)$ measurable, $w^{*}$-l.s.c. in $X^{*}$ and for every $(A, v) \in \Sigma_{0} \times L^{\infty}\left(\Sigma_{0}, X_{w^{*}}^{*}\right)$ we have that

$$
\int_{A} \varphi(\omega, v(\omega)) d \mu(\omega)=\int_{A} \sigma(v(\omega), F(\omega)) d \mu(\omega)
$$

Then we write $\varphi\left(\omega, x^{*}\right)=E^{\Sigma_{0}} \sigma\left(x^{*}, F(\omega)\right)$. Note that since $X$ is separable, $X_{w^{*}}^{*}$ (the Banach space $X^{*}$ equipped with the weak ${ }^{*}$-topology) is a Souslin space and since two comparable Souslin topologies generate the same Borel $\sigma$ field, we have that $B\left(X_{\tau}^{*}\right)=B\left(X_{w^{*}}^{*}\right)$, for any comparable Souslin topology $\tau$ on $X^{*}$.

In the sequel, we will show that the conditional expectation $E^{\Sigma_{0}} \sigma\left(x^{*}, F(\omega)\right)$ exists and is unique up to sets of the form $N \times X^{*}, \mu(N)=0$. We will do this by proving a general representation theorem for nonlinear, local and additive on $\Sigma$ functionals. Recall that $L^{1}(\Omega, X)^{*}=L^{\infty}\left(\Omega, X_{w^{*}}^{*}\right)$ (cf. Ionescu-Tulcea [20]). Here $L^{\infty}\left(\Omega, X_{w^{*}}^{*}\right)$ is the Banach space of all $f: \Omega \rightarrow X^{*}$ which are $w^{*}$-measurable and $\|f(\cdot)\| \in L^{\infty}(\Omega)$.

We start with two auxiliary results that will be needed in the sequel.

Lemma 6. If $L: \Sigma \times L^{\infty}\left(\Omega, X_{w^{*}}^{*}\right) \rightarrow \overline{\mathbb{R}}=\mathbb{R} \cup\{+\infty\}$ is additive on $\Sigma$, then $L$ is local on $\Sigma$ if and only if, for all $A, B \in \Sigma, A \cap B=\varnothing$, we have

$$
L\left(A \cup B, \chi_{A} x^{*}+\chi_{B} y^{*}\right)=L\left(A, x^{*}\right)+L\left(B, y^{*}\right) .
$$

Proof. $\Rightarrow$ : Note that $x^{*}=\chi_{A} x^{*}+\chi_{B} y^{*}$ on $A$ and $y^{*}=\chi_{A} x^{*}+\chi_{B} y^{*}$ on $B$. Since by hypothesis $L$ is local on $\Sigma$, we have $L\left(A, x^{*}\right)=L\left(A, \chi_{A} x^{*}+\chi_{B} y^{*}\right)$ and $L\left(B, y^{*}\right)=L\left(B, \chi_{A} x^{*}+\chi_{B} y^{*}\right)$.

$\Leftarrow$ : Let $x^{*}, y^{*} \in L^{\infty}\left(\Omega, X_{w^{*}}^{*}\right)$ and $x^{*}=y^{*}, \mu$-a.e. on $B \in \Sigma$. Then we have $L\left(B, x^{*}\right)=L\left(B \cup \varnothing, \chi_{B} x^{*}+\chi_{\varnothing} y^{*}\right)=L\left(B, \chi_{B} x^{*}\right)$ and $L\left(B, y^{*}\right)=$ $L\left(B \cup \varnothing, \chi_{B} y^{*}+\chi_{\varnothing} x^{*}\right)=L\left(B, \chi_{B} y^{*}\right)$. Since by hypothesis $x^{*}=y^{*} \mu$-a.e. on $B$, we get $L\left(B, \chi_{B} x^{*}\right)=L\left(B, \chi_{B} y^{*}\right) \Rightarrow L\left(B, x^{*}\right)=L\left(B, y^{*}\right) \Rightarrow L$ is local on $\Sigma$. Q.E.D.

The second auxiliary result is the following:

Lemma 7. If $\varphi_{1}, \varphi_{2}: \Omega \times X^{*} \rightarrow \overline{\mathbb{R}}=\mathbb{R} \cup\{+\infty\}$ are $\Sigma \times B\left(X_{m}^{*}\right)=\Sigma \times B\left(X_{w^{*}}^{*}\right)$ measurable integrands such that for all $\left(A, x^{*}\right) \in \Sigma \times L^{\infty}\left(\Omega, X_{w^{*}}^{*}\right)$ we have

$$
\int_{A} \varphi_{1}\left(\omega, x^{*}(\omega)\right) d \mu(\omega) \leq \int_{A} \varphi_{2}\left(\omega, x^{*}(\omega)\right) d \mu(\omega),
$$

then $\varphi_{1}\left(\omega, x^{*}\right) \leq \varphi_{2}\left(\omega, x^{*}\right)$ for all $\left(\omega, x^{*}\right) \in(\Omega \backslash N) \times X^{*}, \mu(N)=0$.

Proof. Let $\widehat{\Omega}=\left\{\omega \in \Omega: \exists x^{*} \in X^{*}, \varphi_{2}\left(\omega, x^{*}\right)<\infty, \varphi_{1}\left(\omega, x^{*}\right)>\varphi_{2}\left(\omega, x^{*}\right)\right\}$. 
If

$$
\begin{aligned}
\Gamma & =\left\{\left(\omega, x^{*}\right) \in \Omega \times X^{*}: \varphi_{2}\left(\omega, x^{*}\right)<\infty, \varphi_{1}\left(\omega, x^{*}\right)>\varphi_{2}\left(\omega, x^{*}\right)\right\} \\
& =\bigcup_{n \geq 1}\left\{\left(\omega, x^{*}\right) \in \Omega \times X^{*}: \varphi_{2}\left(\omega, x^{*}\right) \leq n, \varphi_{1}\left(\omega, x^{*}\right)>\varphi_{2}\left(\omega, x^{*}\right)\right\} \\
& \in \Sigma \times B\left(X_{w^{*}}^{*}\right) .
\end{aligned}
$$

Since $X_{w^{*}}^{*}$ is a Souslin space, by the von Neumann-Aumann projection theorem (cf. Wagner [44]), we have that $\operatorname{proj}_{\Omega} \Gamma=\widehat{\Omega} \in \Sigma$. Also from Aumann's selection theorem (see Wagner [44, Theorem 5.10]), we can find $y^{*}: \widehat{\Omega} \rightarrow X^{*}$ $w^{*}$-measurable such that $\left(\omega, y^{*}(\omega)\right) \in \Gamma$ for all $\omega \in \widehat{\Omega}$.

Let $\widehat{\Omega}_{n}=\left\{\omega \in \widehat{\Omega}:\left\|y^{*}(\omega)\right\| \leq n, \varphi_{2}\left(\omega, y^{*}(\omega)\right) \leq n, \varphi_{1}\left(\omega, y^{*}(\omega)\right)>\right.$ $\left.\varphi_{2}\left(\omega, y^{*}(\omega)\right)\right\}$. Then $\widehat{\Omega}=\bigcup_{n \geq 1} \widehat{\Omega}_{n}$. If $\mu(\widehat{\Omega})>0$, then, for some $n_{0} \geq 1$, $\mu\left(\widehat{\Omega}_{n_{0}}\right)>0$. Let $v^{*} \in X^{*}$ with $\left\|v^{*}\right\| \leq n_{0}$ and define

$$
z^{*}(\omega)= \begin{cases}y^{*}(\omega) & \text { if } \omega \in \widehat{\Omega}_{n_{0}}, \\ v^{*} & \text { otherwise. }\end{cases}
$$

Then clearly $z^{*}(\cdot) \in L^{\infty}\left(\Omega, X_{w^{*}}^{*}\right)$ and

$$
\int_{\widehat{\Omega}_{n_{0}}} \varphi_{1}\left(\omega, z^{*}(\omega)\right) d \mu(\omega)>\int_{\widehat{\Omega}_{n_{0}}} \varphi_{2}\left(\omega, z^{*}(\omega)\right) d \mu(\omega),
$$

a contradiction to our hypothesis. So $\mu(\widehat{\Omega})=0$; the proof is complete. Q.E.D.

On $X_{w^{*}}^{*}$ we can define a metric topology induced by the metric

$$
\delta\left(x^{*}, y^{*}\right)=\sum_{n \geq 1} \frac{1}{2^{n}} \frac{\left|\left(x_{n}, x^{*}-y^{*}\right)\right|}{1+\left|\left(x_{n}, x^{*}-y^{*}\right)\right|}
$$

where $\left\{x_{n}\right\}_{n \geq 1}$ is a countable dense subset of $X$. It is well known that the $\delta$ metric topology, the weak ${ }^{*}$-topology and the topology of uniform convergence on compacta (compact convergence topology $\tau_{c}$; cf. Schaefer [42]) all coincide on closed bounded subsets of $X^{*}$. Of course by the Banach-Dieudonne theorem (see Schaefer [42, p. 151]), the $\delta$-metric topology is weaker than $\tau_{c}$. Also $\delta(\cdot, \cdot)$ induces a metric $\hat{\delta}(\cdot, \cdot)$ on $L^{\infty}\left(\Omega, X_{w^{*}}^{*}\right)$ defined by

$$
\hat{\delta}\left(x^{*}, y^{*}\right)=\int_{\Omega} \delta\left(x^{*}(\omega), y^{*}(\omega)\right) d \mu(\omega) .
$$

Now we are ready for our representation theorem:

Theorem 8. If $L: \Sigma \times L^{\infty}\left(\Omega, X_{w^{*}}^{*}\right) \rightarrow \overline{\mathbb{R}}_{+}=\mathbb{R}_{+} \cup\{+\infty\}$ is a functional such that

(1) $L(\cdot, \cdot)$ is local on $\Sigma$,

(2) $L(\cdot, \cdot)$ is additive on $\Sigma$,

(3) there exists $x_{0}^{*} \in L^{\infty}\left(\Omega, X_{w^{*}}^{*}\right)$ such that $A \rightarrow L\left(A, x_{0}^{*}\right)$ is a finite measure which is absolutely continuous with respect to $\mu(\cdot)$,

(4) $x^{*} \rightarrow L\left(A, x^{*}\right)$ is $\hat{\delta}$-l.s.c., then there exists an integrand $\varphi: \Omega \times X^{*} \rightarrow \overline{\mathbb{R}}_{+}$which is proper, $\Sigma \times$ $B\left(X_{\delta}^{*}\right)=\Sigma \times B\left(X_{w^{*}}^{*}\right)$-measurable, for every $\omega \in \Omega, \varphi(\omega, \cdot)$ is $\delta$ l.s.c. on $X^{*}$ and for all $\left(A, x^{*}\right) \in \Sigma \times L^{\infty}\left(\Omega, X_{w^{*}}^{*}\right), \quad L\left(A, x^{*}\right)=$ 
$\int_{A} \varphi\left(\omega, x^{*}(\omega)\right) d \mu(\omega)$. Furthermore, $\varphi\left(\omega, x^{*}\right)$ is unique up to sets $N \times X^{*}, \mu(N)=0$.

Proof. Let $L_{n}: \Sigma \times L^{\infty}\left(\Omega, X_{w^{*}}^{*}\right) \rightarrow \overline{\mathbb{R}}_{+}$be defined by

$$
L_{n}\left(A, x^{*}\right)=\inf \left[L\left(A, y^{*}\right)+n \hat{\delta}\left(\chi_{A} x^{*}, \chi_{A} y^{*}\right): y^{*} \in L^{\infty}\left(\Omega, X_{w^{*}}^{*}\right)\right] .
$$

Then clearly for every $\left(A, x^{*}\right) \in \Sigma \times L^{\infty}\left(\Omega, X_{w^{*}}^{*}\right)$ we have $0 \leq L_{n}\left(A, x^{*}\right) \leq$ $L\left(A, x^{*}\right)$ and $L_{n}\left(A, x^{*}\right) \uparrow L\left(A, x^{*}\right)$ as $n \rightarrow \infty$.

First we will show that, for each $n \geq 1, A \rightarrow L_{n}(A, u)$ is additive. To this end let $A, B \in \Sigma$ with $A \cap B=\varnothing$. We have:

(1)

$$
\begin{aligned}
L_{n}\left(A \cup B, x^{*}\right)= & \inf \left[L\left(A \cup B, y^{*}\right)+n \hat{\delta}\left(\chi_{A \cup B} x^{*}, \chi_{A \cup B} y^{*}\right): y^{*} \in L^{\infty}\left(\Omega, x_{w^{*}}^{*}\right)\right] \\
= & \inf \left[L\left(A \cup B, y^{*}\right)+n \hat{\delta}\left(\chi_{A} x^{*}, \chi_{A} y^{*}\right)\right. \\
& \left.+n \hat{\delta}\left(\chi_{B} x^{*}, \chi_{B} y^{*}\right): y^{*} \in L^{\infty}\left(\Omega, X_{w^{*}}^{*}\right)\right] \\
\geq & \inf \left[L\left(A \cup B, y^{*}\right)+n \hat{\delta}\left(\chi_{A} x^{*}, \chi_{A} y^{*}\right): y^{*} \in L^{\infty}\left(\Omega, X_{w^{*}}^{*}\right)\right] \\
& +\inf \left[L\left(A \cup B, y^{*}\right)+n \hat{\delta}\left(\chi_{B} x^{*}, \chi_{B} y^{*}\right): y^{*} \in L^{\infty}\left(\Omega, X_{w^{*}}^{*}\right)\right] \\
= & L_{n}\left(A, x^{*}\right)+L_{n}\left(B, x^{*}\right) .
\end{aligned}
$$

On the other hand, let $\varepsilon>0$. Then we can find $y_{1}^{*}, y_{2}^{*} \in L^{\infty}\left(\Omega, X_{w^{*}}^{*}\right)$ such that

$$
L\left(A, y_{1}^{*}\right)+n \hat{\delta}\left(\chi_{A} x^{*}, \chi_{A} y_{1}^{*}\right) \leq L_{n}\left(A, x^{*}\right)+\frac{\varepsilon}{2}
$$

and

$$
L\left(B, y_{2}^{*}\right)+n \hat{\delta}\left(\chi_{B} x^{*}, \chi_{B} y_{2}^{*}\right) \leq L_{n}\left(B, x^{*}\right)+\frac{\varepsilon}{2} .
$$

Adding these two inequalities, we get

$$
\begin{aligned}
& L\left(A, y_{1}^{*}\right)+L\left(B, y_{2}^{*}\right)+n \hat{\delta}\left(\chi_{A} x^{*}, \chi_{A} y_{1}^{*}\right)+n \hat{\delta}\left(\chi_{B} x^{*}, \chi_{B} y_{2}^{*}\right) \\
& \leq L_{n}\left(A, x^{*}\right)+L_{n}\left(B, x^{*}\right)+\varepsilon \\
& \Rightarrow L\left(A \cup B, \chi_{A} y_{1}^{*}+\chi_{B} y_{2}^{*}\right)+n \hat{\delta}\left(\chi_{A \cup B} x^{*}, \chi_{A \cup B}\left(\chi_{A} y_{1}^{*}+\chi_{B} y_{2}^{*}\right)\right) \\
& \leq L_{n}\left(A, x^{*}\right)+L_{n}\left(B, x^{*}\right)+\varepsilon \quad(\mathrm{cf} . \text { Lemma 6) } \\
& \Rightarrow L_{n}\left(A \cup B, x^{*}\right) \leq L_{n}\left(A, x^{*}\right)+L_{n}\left(B, x^{*}\right)+\varepsilon .
\end{aligned}
$$

Let $\varepsilon \downarrow 0$, to get that

$$
L_{n}\left(A \cup B, x^{*}\right) \leq L_{n}\left(A, x^{*}\right)+L_{n}\left(B, x^{*}\right) .
$$

From (1) and (2) above, we conclude that $A \rightarrow L_{n}\left(A, x^{*}\right)$ is indeed additive. Furthermore $0 \leq L_{n}\left(A, x^{*}\right) \leq L\left(A, x_{0}^{*}\right)+n \hat{\delta}\left(\chi_{A} x^{*}, \chi_{A} x_{0}^{*}\right)$. But because of hypothesis (3) $A \rightarrow L\left(A, x_{0}^{*}\right)+n \hat{\delta}\left(\chi_{A} x^{*}, \chi_{A} x_{0}^{*}\right)$ is a finite measure. So from Theorem 1.2.8, p. 11 of Ash [2], we deduce that $A \rightarrow L_{n}\left(A, x^{*}\right)$ is a measure on $\Sigma$. In addition, since by hypothesis $L\left(\cdot, x_{0}^{*}\right) \ll \mu$ we have $L_{n}\left(\cdot, x^{*}\right) \ll \mu$ for every $x^{*} \in L^{\infty}\left(\Omega, X_{w^{*}}^{*}\right)$ and every $n \geq 1$. Apply the Radon-Nikodym theorem to get that for every $x^{*} \in X^{*}$ there exists $\varphi_{n}\left(\cdot, x^{*}\right) \in L^{1}(\Omega), \varphi_{n}\left(\omega, x^{*}\right) \geq 0$ $\mu$-a.e. such that

$$
L_{n}\left(A, x^{*}\right)=\int_{A} \varphi_{n}\left(\omega, x^{*}\right) d \mu(\omega)
$$

for all $\left(A, x^{*}\right) \in \Sigma \times X^{*}$. Note that for all $n \geq 1$, since

$$
\left|L_{n}\left(A, x^{*}\right)-L_{n}\left(A, y^{*}\right)\right| \leq n \hat{\delta}\left(x^{*}, y^{*}\right)
$$


we have

$$
\begin{aligned}
& \int_{A}\left|\varphi_{n}\left(\omega, x^{*}\right)-\varphi_{n}\left(\omega, y^{*}\right)\right| d \mu(\omega) \leq n \mu(A) \delta\left(x^{*}, y^{*}\right) \\
& \quad \leq n \delta\left(x^{*}, y^{*}\right) \quad \text { for all } A \in \Sigma \\
& \quad \Rightarrow\left|\varphi_{n}\left(\omega, x^{*}\right)-\varphi_{n}\left(\omega, y^{*}\right)\right| \leq n \delta\left(x^{*}, y^{*}\right)
\end{aligned}
$$

for all $\omega \in \Omega \backslash N, \mu(N)=0$ and all $x^{*}, y^{*} \in D^{*}$, where $D^{*}$ is a countable subset of $X^{*}$ dense for the $\delta$-metric. Then for all $\omega \in \Omega \backslash N$, we can extend $\varphi_{n}(\omega, \cdot)$ on all of $X^{*}$ and have $\left|\varphi_{n}\left(\omega, x^{*}\right)-\varphi_{n}\left(\omega, y^{*}\right)\right| \leq n \delta\left(x^{*}, y^{*}\right)$ for all $\omega \in \Omega \backslash N, \mu(N)=0$. Set $\varphi_{n}\left(\omega, x^{*}\right)$ for $\omega \in N, \mu(N)=0$. Now if $x^{*}(\omega)=\sum_{k=1}^{N} \chi_{B_{k}}(\omega) v_{k}^{*}$ with $B_{k} \in \Sigma, v_{k}^{*} \in X^{*}$, then from Lemma 6 we get

$$
\begin{aligned}
L_{n}\left(A, x^{*}\right) & =L_{n}\left(A, \sum_{k=1}^{N} \chi_{B_{k}} v_{k}^{*}\right)=\sum_{k=1}^{N} L_{n}\left(A \cap B_{k}, v_{k}^{*}\right) \\
& =\sum_{k=1}^{N} \int_{A \cap B_{k}} \varphi_{n}\left(\omega, v_{k}^{*}\right) d \mu(\omega)=\int_{A} \varphi_{n}\left(\omega, x^{*}(\omega)\right) d \mu(\omega) .
\end{aligned}
$$

But simple functions are dense in $\left(L^{\infty}\left(\Omega, X_{w^{*}}^{*}\right), \hat{\delta}\right)$. Hence exploiting the $\hat{\delta}$-continuity of $L_{n}(A, \cdot)$ and the $\delta$-continuity of $\varphi_{n}(\omega, \cdot)$, we get that for all $\left(A, x^{*}\right) \in \Sigma \times L^{\infty}\left(\Omega, X_{w^{*}}^{*}\right)$ we have

$$
L_{n}\left(A, x^{*}\right)=\int_{A} \varphi_{n}\left(\omega, x^{*}(\omega)\right) d \mu(\omega) .
$$

Next set $\varphi\left(\omega, x^{*}\right)=\sup _{n \geq 1} \varphi_{n}\left(\omega, x^{*}\right)$. So $\left(\omega, x^{*}\right) \rightarrow \varphi\left(\omega, x^{*}\right)$ is $\Sigma \times$ $B\left(X_{\delta}^{*}\right)=\Sigma \times B\left(X_{w^{*}}^{*}\right)$-measurable and $x^{*} \rightarrow \varphi\left(\omega, x^{*}\right)$ is $\delta$-l.s.c. In addition from the monotone convergence theorem, we get

$$
\begin{aligned}
L\left(A, x^{*}\right) & =\sup _{n \geq 1} L_{n}\left(A, x^{*}\right)=\sup _{n \geq 1} \int_{A} \varphi_{n}\left(\omega, x^{*}(\omega)\right) d \mu(\omega) \\
& =\int_{A} \varphi\left(\omega, x^{*}(\omega)\right) d \mu(\omega)
\end{aligned}
$$

for all $\left(A, x^{*}\right) \in \Sigma \times L^{\infty}\left(\Omega, X_{w^{*}}^{*}\right)$.

Finally uniqueness of $\varphi\left(\omega, x^{*}\right)$ up to sets $N \times X^{*}, \mu(N)=0$, follows immediately from Lemma 7. Q.E.D.

Remark. It is easy to see that, by using Lemma 7 , we can have that $\varphi(\omega, \cdot)$ is convex (resp. sublinear), provided $L(A, \cdot)$ is.

We can now use this representation result to relate the $\Sigma_{0}$-conditional expectation of $\sigma\left(x^{*}, F(\omega)\right)$ and the support function $\sigma\left(x^{*}, E^{\Sigma_{0}} F(\omega)\right)$. Our result improves a similar result of Papageorgiou [32], where $X^{*}$ is assumed to be separable and $F(\cdot)$ integrably bounded (see also Wang-Xue [45]).

Theorem 9. If $F: \Omega \rightarrow P_{f}(X)$ is a measurable and $S_{F}^{1} \neq \varnothing$, then the $\Sigma_{0}$-conditional expectation of $\sigma\left(x^{*}, F(\omega)\right)$ exists, is unique up to sets $N \times X^{*}, \mu(N)=0$ and for all $\omega \in \Omega \backslash N, \mu(N)=0$ and all $x^{*} \in X^{*}$

$$
E^{\Sigma_{0}} \sigma\left(x^{*}, F(\omega)\right)=\sigma\left(x^{*}, E^{\Sigma_{0}} F(\omega)\right) \text {. }
$$

Proof. Let $f \in S_{F}^{1}$. Then for every $\omega \in \Omega \backslash N^{\prime}, \mu\left(N^{\prime}\right)=0$ and every $x^{*} \in X^{*}$, we have

$$
\left(x^{*}, f(\omega)\right) \leq \sigma\left(x^{*}, F(\omega)\right)
$$


Define $L: \Sigma_{0} \times L^{\infty}\left(\Sigma_{0}, X_{w^{*}}^{*}\right) \rightarrow \overline{\mathbb{R}}_{+}$by

$$
L\left(A, x^{*}\right)=\int_{A}\left[\sigma\left(x^{*}(\omega), F(\omega)\right)-\left(x^{*}(\omega), f(\omega)\right)\right] d \mu(\omega) .
$$

Note that $m(A, 0)=0$ for all $A \in \Sigma_{0}$. Also we claim that if $A \in \Sigma_{0}$ for every $\lambda \in \mathbb{R}$, the set

$$
\Gamma_{\lambda}^{A}=\left\{x^{*} \in L^{\infty}\left(\Sigma_{0}, X_{w^{*}}^{*}\right): L\left(A, x^{*}\right) \leq \lambda\right\}
$$

is $\hat{\delta}$-closed. Indeed if $x_{n}^{*} \stackrel{\hat{\delta}}{\rightarrow} x^{*}$ and $x_{n}^{*} \in \Gamma_{\lambda}^{A}, n \geq 1$, then by passing to a subsequence if necessary, we may assume that $\delta\left(x_{n}^{*}(\omega), x^{*}(\omega)\right) \rightarrow 0 \mu$-a.e. So for almost all $\omega \in \Omega,\left\{x_{n}^{*}(\omega)\right\}_{n \geq 1}$ is in a $w^{*}$-bounded set in $X^{*}$, hence is a bounded set in $X^{*}$. But on such sets the $w^{*}$-topology and the $\delta$-metric topology coincide. So $x_{n}^{*}(\omega) \stackrel{w^{*}}{\rightarrow} x^{*}(\omega) \mu$-a.e. So applying Fatou's lemma, we get

$$
\begin{aligned}
m\left(A, x^{*}\right) & =\int_{A}\left[\sigma\left(x^{*}(\omega), F(\omega)\right)-\left(x^{*}(\omega), f(\omega)\right)\right] d \mu(\omega) \\
& \leq \underline{\lim } \int_{A}\left[\sigma\left(x_{n}^{*}(\omega), F(\omega)\right)-\left(x_{n}^{*}(\omega), f(\omega)\right)\right] d \mu(\omega) \leq \lambda \\
& \Rightarrow \Gamma_{\lambda}^{A} \text { is } \hat{\delta} \text {-closed } \\
& \Rightarrow L(A, \cdot) \text { is } \hat{\delta} \text {-l.s.c. }
\end{aligned}
$$

Apply Theorem 8 (with $x_{0}^{*}(\cdot) \equiv 0$ ), to get $\varphi: \Omega \times X^{*} \rightarrow \overline{\mathbb{R}}_{+}$a $\Sigma_{0} \times B\left(X_{w^{*}}^{*}\right)$ measurable integrand, which is $\delta$-l.s.c. in $x^{*}$ and for all $\left(A, x^{*}\right) \in \Sigma_{0} \times$ $L^{\infty}\left(\Sigma_{0}, X^{*}\right)$

$$
L\left(A, x^{*}\right)=\int_{A} \varphi\left(\omega, x^{*}(\omega)\right) d \mu(\omega)
$$

and $\varphi\left(w, x^{*}\right)$ is unique up to sets $N \times X^{*}, \mu(N)=0$. So

$$
\int_{A}\left[\sigma\left(x^{*}(\omega), F(\omega)\right)-\left(x^{*}(\omega), f(\omega)\right)\right] d \mu(\omega)=\int_{A} \varphi\left(\omega, x^{*}(\omega)\right) d \mu(\omega) .
$$

So by definition $E^{\Sigma_{0}} \sigma\left(x^{*}, F(\omega)\right)=\varphi\left(\omega, x^{*}\right)+\left(x^{*}, E^{\Sigma_{0}} f(\omega)\right)$ for all $\omega \epsilon$ $\Omega \backslash N, \mu(N)=0$ and all $x^{*} \in X^{*}$. On the other hand, from Theorem 2.2 of Hiai-Umegaki [17] and the definition of the set-valued conditional expectation, we have that

$$
\begin{aligned}
\int_{A} \sigma\left(x^{*}(\omega), F(\omega)\right) d \mu(\omega)=\int_{A} \sigma\left(x^{*}(\omega), E^{\Sigma_{0}} F(\omega)\right) d \mu(\omega) \\
\quad \Rightarrow \int_{A}\left[\varphi\left(\omega, x^{*}(\omega)\right)+\left(x^{*}(\omega), E^{\Sigma_{0}} f(\omega)\right)\right] d \mu(\omega) \\
\quad=\int_{A} \sigma\left(x^{*}(\omega), E^{\Sigma_{0}} F(\omega)\right) d \mu(\omega) \\
\Rightarrow E^{\Sigma_{0}} \sigma\left(x^{*}, F(\omega)\right)=\sigma\left(x^{*}, E^{\Sigma_{0}} F(\omega)\right) \text { for all } \omega \in \Omega \backslash N, \mu(N)=0
\end{aligned}
$$

and all $x^{*} \in X^{*}$ (cf. Lemma 7). Q.E.D.

We can use this result to characterize the integrable selectors of $E^{\Sigma_{0}} F(\cdot)$. 
Proposition 10. If $X^{*}$ is separable, $F: \Omega \rightarrow P_{f c}(X)$ is an integrable bounded multifunction and $g \in L^{1}\left(\Sigma_{0}, X\right)$, then $g \in S_{E^{\Sigma_{0} F}}^{1}$ if and only if $\int_{A} g(\omega) d \mu(\omega)$ $\in \operatorname{cl} \int_{A} F(\omega) d \mu(\omega)$ for all $A \in \Sigma_{0}$.

Proof. $\Rightarrow$ : Follows immediately from Theorem $5.4\left(2^{\circ}\right)$ of Hiai-Umegaki [17].

$\Leftarrow$ : Using Theorem 9 above, we see that for every $x^{*} \in X^{*}$

$$
\begin{gathered}
\int_{A}\left(x^{*}, g(\omega)\right) d \mu(\omega) \leq \int_{A} \sigma\left(x^{*}, F(\omega)\right) d \mu(\omega)=\int_{A} \sigma\left(x^{*}, E^{\Sigma_{0}} F(\omega)\right) d \mu(\omega) \\
\Rightarrow\left(x^{*}, g(\omega)\right) \leq \sigma\left(x^{*}, E^{\Sigma_{0}} F(\omega)\right)
\end{gathered}
$$

for all $\omega \in \Omega \backslash N, \mu(N)=0$ and all $x^{*} \in D^{*} \subseteq X^{*}$ a countable strongly dense subset. Recall that $E^{\Sigma_{0}} F(\cdot)$ is integrably bounded and so $E^{\Sigma_{0}} F(\omega)$ is $\mu$-a.e. bounded. So $\sigma\left(\cdot, E^{\Sigma_{0}} F(\omega)\right)$ is $\mu$-a.e. strongly continuous. Hence we deduce that

$$
\left(x^{*}, g(\omega)\right) \leq \sigma\left(x^{*}, E^{\Sigma_{0}} F(\omega)\right)
$$

for all $\omega \in \Omega \backslash N^{\prime}, \mu\left(N^{\prime}\right)=0$ and all $x^{*} \in X^{*}$. Since $E^{\Sigma_{0}} F(\cdot)$ is $P_{f c}(X)$ valued, we conclude that $g(\omega) \in E^{\Sigma_{0}} F(\omega) \mu$-a.e. $\Rightarrow g \in S_{E^{\Sigma_{0} F}}^{1}$. Q.E.D.

We can drop the separability hypothesis on $X^{*}$ if we strengthen our hypothesis on $F(\cdot)$.

Proposition 11. If $F: \Omega \rightarrow P_{w k c}(X)$ is integrably bounded and $g \in L^{1}\left(\Sigma_{0}, X\right)$, then $g \in S_{E^{\Sigma_{0} F}}^{1}$ if and only if $\int_{A} g(\omega) d \mu(\omega) \in \int_{A} F(\omega) d \mu(\omega)$ for all $A \in \Sigma_{0}$. Proof. The proof is the same as that of Proposition 10, only now we use the fact that $E^{\Sigma_{0}} F(\omega) \in P_{w k c}(X) \mu$-a.e. (cf. Proposition 3) and so $\sigma\left(\cdot, E^{\Sigma_{0}} F(\omega)\right.$ ) is $\mu$-a.e. $m\left(X^{*}, X\right)$-continuous (here $m\left(X^{*}, X\right)$ denotes the Mackey topology on $X^{*}$, defined by the dual pair $\left(X^{*}, X\right)$ ). Also note that since $X$ is separable, $\left(X^{*}, m\right)$ is separable (see for example Wilansky [46, p. 144]). Q.E.D.

\section{OPTIONAL SAMPLING}

In this section we prove an optimal sampling theorem for set-valued martingales, extending an earlier result of Alo-de Korvin-Roberts [1]. Here $\left\{\Sigma_{n}\right\}_{n \geq 1}$ is an increasing sequence of sub- $\sigma$-fields of $\Sigma$ such that $\Sigma=\sigma\left(\bigcup_{n \geq 1} \Sigma_{n}\right)$.

Theorem 12. If $\left\{F_{n}(\cdot)\right\}_{n \geq 1} \subseteq \mathscr{L}_{f c}^{1}(X),\left\{F_{n}, \Sigma_{n}\right\}_{n \geq 1}$ is a set-valued martingale, $\left\{\tau_{m}\right\}_{m \geq 1} \subseteq T$ is an increasing sequence of stopping times, $\widehat{F}_{m}=F_{\tau_{m}}$ and $\widehat{\Sigma}_{m}=$ $\Sigma_{\tau_{m}}$,

then $\left\{\widehat{F}_{m}, \widehat{\Sigma}_{n}\right\}_{m \geq 1}$ is a set-valued martingale.

Proof. From Luu [25], we know that, for each $n \geq 1$, we can find $\left\{f_{n}^{k}(\cdot)\right\}_{k \geq 1} \subseteq$ $S_{F_{n}}^{1}$ such that $F_{n}(\omega)=\operatorname{cl}\left\{f_{n}^{k}(\omega)\right\}_{k \geq 1}$ for all $\omega \in \Omega$ and for each $k \geq 1$, $\left\{f_{n}^{k}(\cdot), \Sigma_{n}\right\}_{n \geq 1}$ if a vector-valued martingale. Invoking Theorem V.1.8, p. 129 of Egghe [15], we know that $\left\{f_{\tau_{m}}^{k}=\hat{f}_{m}^{k}, \widehat{\Sigma}_{m}\right\}_{n \geq 1}$ is a vector-valued martingale and of course $\widehat{F}_{m}(\omega)=\operatorname{cl}\left\{\hat{f}_{m}^{k}(\omega)\right\}_{k \geq 1}$ for all $\omega \in \Omega$. So from Corollary 2.3 of Luu [25], we conclude that $\left\{\widehat{F}_{m}, \widehat{\Sigma}_{m}\right\}_{m \geq 1}$ is a set-valued martingale. Q.E.D. 


\section{SET-VAlued AMARTS AND UNIFORM AMARTS}

In [6] (pp. 189-190) Bellow proved that every point-valued quasi-martingale is a uniform amart. The next proposition shows that the same holds true for random sets.

Proposition 13. If the adopted sequence $\left\{F_{n}, \Sigma_{n}\right\}_{n \geq 1}$ is a set-valued quasimartingale in $\mathscr{L}_{f c}^{1}(X)$, then $\left\{F_{n}, \Sigma_{n}\right\}_{n \geq 1}$ is a set-valued uniform amart.

Proof. From Proposition 1.6 of Luu [25] (see also Theorem 6.1 of [34]), we know that there exists a martingale $\left\{M_{n}, \Sigma_{n}\right\}_{n \geq 1}$ in $\mathscr{L}_{f_{c}}^{1}(X)$ such that

$$
\Delta\left(F_{n}, M_{n}\right) \rightarrow 0 \text { and } \Delta\left(E^{\Sigma_{m}} F_{n}, M_{m}\right) \rightarrow 0 \text { as } m \rightarrow \infty, n \geq m, m \geq 1 .
$$

Let $k \in \mathbb{N}_{+}$and $\tau \in T$ be given such that $\tau \geq k$ and let $v$ be any positive integer such that $v \geq \tau \geq k$. Then we can find $m>v$ such that

Furthermore we have

$$
\Delta\left(E^{\Sigma_{i}} F_{m}, M_{m}\right) \leq \frac{1}{v 2^{k}}, \quad k \leq i \leq v .
$$

$$
\begin{aligned}
\Delta\left(F_{\tau}, M_{\tau}\right) & =\sum_{i=k}^{v} \int_{\{\tau=i\}} h\left(F_{i}, M_{i}\right) d \mu \\
& \leq \sum_{i=k}^{v} \int_{\{\tau=i\}}\left(h\left(F_{i}, E^{\Sigma_{i}} F_{m}\right)+h\left(E^{\Sigma_{i}} F_{m}, M_{i}\right)\right) d \mu \\
& =\sum_{i=k}^{v} \int_{\{\tau=i\}} h\left(F_{i}, E^{\Sigma_{i}} F_{m}\right) d \mu+\sum_{i=k}^{v} \Delta\left(E^{\Sigma_{i}} F_{m}, M_{i}\right) \\
& \leq \sum_{i=k}^{v} \int_{\{\tau=i\}} h\left(F_{i}, E^{\Sigma_{i}} F_{m}\right) d \mu+\frac{1}{2^{k}} .
\end{aligned}
$$

Note that for each $i=k, \ldots, v$ we have

$$
\begin{aligned}
\int_{\{\tau=i\}} h\left(F_{i}, E^{\Sigma_{i}} F_{m}\right) d \mu=\int_{\{\tau=i\}} h\left(E^{\Sigma_{i}} F_{i}, E^{\Sigma_{i}} E^{\Sigma_{m-1}} F_{m}\right) d \mu \\
\leq \int_{\{\tau=i\}} E^{\Sigma_{i}} h\left(F_{i}, E^{\Sigma_{m-1}} F_{m}\right) d \mu \quad \text { (see Papageorgiou [34, p. 141]) } \\
=\int_{\{\tau=i\}} h\left(F_{i}, E^{\Sigma_{m-1}} F_{m}\right) d \mu \quad\left(\text { since }\{\tau=i\} \in \Sigma_{i}\right) \\
\leq \sum_{j=k}^{m-1} \int_{\{\tau=i\}} h\left(F_{j}, E^{\Sigma_{j}} F_{j+1}\right) d \mu \quad \text { (triangle inequality). }
\end{aligned}
$$

Therefore we have

$$
\begin{aligned}
& \Delta\left(F_{\tau}, M_{\tau}\right) \leq \sum_{i=k}^{v} \sum_{j=k}^{m-1} \int_{\{\tau=i\}} h\left(F_{j}, E^{\Sigma_{j}} F_{j+1}\right) d \mu+\frac{1}{2^{k}} \\
& =\sum_{j=k}^{m-1} \Delta\left(F_{j}, E^{\Sigma_{j}} F_{j+1}\right)+\frac{1}{2^{k}} \\
& \Rightarrow \lim _{\tau \in T} \Delta\left(F_{\tau}, M_{\tau}\right) \leq \lim _{m \geq \tau \geq k \rightarrow \infty} \sum_{j=k}^{m-1} \Delta\left(F_{j}, E^{\Sigma_{j}} F_{j+1}\right)+\lim _{k \rightarrow \infty} \frac{1}{2^{k}}=0 .
\end{aligned}
$$


So finally we have for $\sigma \geq \tau, \sigma, \tau \in T$ :

$$
\begin{aligned}
& \Delta\left(F_{\tau}, E^{\Sigma_{\tau}} F_{\sigma}\right) \leq \Delta\left(F_{\tau}, M_{\tau}\right)+\Delta\left(M_{\tau}, E^{\Sigma_{\tau}} M_{\sigma}\right)+\Delta\left(E^{\Sigma_{\tau}} M_{\sigma}, E^{\Sigma_{\tau}} F_{\sigma}\right) \\
& \leq \Delta\left(F_{\tau}, M_{\tau}\right)+\Delta\left(M_{\sigma}, F_{\sigma}\right) \quad(\text { cf. Theorem 12) } \\
& \Rightarrow \lim _{\tau \in T} \sup _{\sigma \geq \tau} \Delta\left(F_{\tau}, E^{\Sigma_{\tau}} F_{\sigma}\right)=0 \\
& \Rightarrow\left\{F_{n}, \Sigma_{n}\right\}_{n \geq 1} \text { is indeed a set-valued amart. Q.E.D. }
\end{aligned}
$$

Remark. Given that a set-valued martingale is easily seen to be a set-valued quasi-martingale, hence by Proposition 13 a set-valued uniform amart, we realize that the convergence results of this section extend those on set-valued martingales existing in the literature. In particular, it extends the recent interesting martingale convergence results of Wang-Xue [45] (section 3).

We start with two convergence theorems for set-valued uniform amarts. The first extends Theorem 2 of Daures [10] and Theorem 6.1 of Hiai [18], which deal with set-valued martingales in $\mathbb{R}^{n}$. It also extends Theorem 3.4 of [34] and Theorem 3.2 of the recent paper of Dam [9].

Theorem 14. If $X^{*}$ is separable and $F_{n}: \Omega \rightarrow P_{f c}(X)$ are $\Sigma_{n}$-measurable multifunctions such that

(1) $\left\{F_{n}, \Sigma_{n}\right\}_{n \geq 1}$ is a set-valued uniform amart,

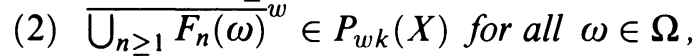

(3) $\left\{\left|F_{n}\right|\right\}_{n \geq 1}$ is uniformly integrable,

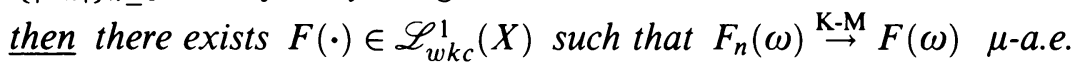

Proof. From Corollary 1.2 of Luu [25], we know that there exists a sequence $\left\{f_{n}^{k}\right\}_{k, n \geq 1}$ such that, for every $k \geq 1,\left\{f_{n}^{k}, \Sigma_{n}\right\}_{n \geq 1}$ is a uniform amart selection of $F_{n}(\cdot)$ (i.e. for every $\omega \in \Omega, f_{n}^{k}(\omega) \in F_{n}(\omega)$ ) and for every $n \geq 1$, ${\overline{\left\{f_{n}^{k}(\omega)\right\}_{k \geq 1}}}=F_{n}(\omega)$. From the Riesz decomposition theorem for vector-valued uniform amarts (see for example Egghe [15, Theorem V.1.4, p. 125]), we have that

$$
f_{n}^{k}(\omega)=m_{n}^{k}(\omega)+p_{n}^{k}(\omega)
$$

with $\left\{m_{n}^{k}, \Sigma_{n}\right\}_{n \geq 1}$ being an $X$-valued martingale and $\left\{p_{n}^{k}, \Sigma_{n}\right\}_{n \geq 1}$ being an $X$-valued uniform potential such that $\left\|p_{n}^{k}(\omega)\right\| \leq s_{n}(\omega) \quad \mu$-a.e. for all $k \geq 1$, with $\left\{s_{n}, \Sigma_{n}\right\}_{n \geq 1}$ being a positive uniform potential (see Luu [25, Theorem 2.1]) and $p_{n}^{k}(\omega) \rightarrow 0 \quad \mu$-a.e. in $X$ as $n \rightarrow \infty$ for every $k \geq 1$. Then for $\mu$-almost all $\omega \in \Omega$, we have

$$
\begin{aligned}
m_{n}^{k}(\omega) & \in \bigcup_{n \geq 1}\left[f_{n}^{k}(\omega)-p_{n}^{k}(\omega)\right] \\
& \subseteq \bigcup_{n \geq 1} f_{n}^{k}(\omega)-\bigcup_{n \geq 1} p_{n}^{k}(\omega)
\end{aligned}
$$

So we can apply Chatterji's convergence theorem (see Egghe [15, p. 57]) and get that

$$
m_{n}^{k}(\omega) \rightarrow m^{k}(\omega) \mu \text {-a.e. in } X \text { as } n \rightarrow \infty \text {. }
$$

Set $F(\omega)=\overline{\operatorname{conv}}\left\{m^{k}(\omega)\right\}_{k \geq 1}, \omega \in \Omega$. Our claim is that this is the desired limit multifunction. First note that because of (3) and the Krein-Smulian 
theorem, we have that $F \in \mathscr{L}_{w k c}^{1}(X)$. Next for $x^{*} \in X^{*}$, we have

$$
\begin{gathered}
\sigma\left(x^{*}, F_{n}(\omega)\right) \leq \sup _{k \geq 1}\left(x^{*}, m_{n}^{k}(\omega)\right)+\sup _{k \geq 1}\left(x^{*}, p_{n}^{k}(\omega)\right) \\
\leq \sup _{k \geq 1}\left(x^{*}, m_{n}^{k}(\omega)\right)+\left\|x^{*}\right\| s_{n}(\omega), \quad \omega \in \Omega .
\end{gathered}
$$

Recall that $\left\{s_{n}, \Sigma_{n}\right\}_{n \geq 1}$ is a positive uniform potential. So by definition $\lim _{\tau \in T} \int_{\Omega} s_{\tau} d \mu=0$. But then by Theorem 2 of Austin-Edgar-Tulcea [4], we have $s_{n}(\omega) \rightarrow 0 \mu$-a.e. Also if $D^{*} \subseteq X^{*}$ is a countable dense subset of $X^{*}$, from Lemma 4 of Neveu [27], we have that for all $\left(\omega, x^{*}\right) \in(\Omega \backslash N) \times D^{*}$, $\mu(N)=0$,

$$
\begin{aligned}
& \sup _{k \geq 1}\left(x^{*}, m_{n}^{k}(\omega)\right) \rightarrow \sup _{k \geq 1}\left(x^{*}, m^{k}(\omega)\right) \\
& \quad \Rightarrow \lim \sigma\left(x^{*}, F_{n}(\omega)\right)=\sigma\left(x^{*}, F(\omega)\right) .
\end{aligned}
$$

Let $x^{*} \in X^{*}$ and let $\left\{x_{m}^{*}\right\}_{m \geq 1} \subseteq D^{*}$ be such that $x_{m}^{*} \rightarrow x^{*}$ in $X^{*}$ as $m \rightarrow$ $\infty$. We have $\sigma\left(x_{m}^{*}, F_{n}(\omega)\right) \rightarrow \sigma\left(x_{m}^{*}, F(\omega)\right)$ as $n \rightarrow \infty$ for all $m \geq 1$ and all $\omega \in \Omega \backslash N$ and $\sigma\left(x_{m}^{*}, F(\omega)\right) \rightarrow \sigma\left(x^{*}, F(\omega)\right)$ as $m \rightarrow \infty$, since $\sigma(\cdot, F(\omega))$ is continuous. that

So we can find a sequence $n \rightarrow m(n)$ not necessarily strictly increasing such

$$
\sigma\left(x_{m(n)}^{*}, F_{n}(\omega)\right) \rightarrow \sigma\left(x^{*}, F(\omega)\right) \text { as } n \rightarrow \infty, \text { for all } \omega \in \Omega \backslash N, \mu(N)=0
$$

(cf. Attouch [3]). Then we have

$$
\begin{aligned}
& \left|\sigma\left(x^{*}, F_{n}(\omega)\right)-\sigma\left(x^{*}, F(\omega)\right)\right| \\
& \quad \leq\left|\sigma\left(x^{*}, F_{n}(\omega)\right)-\sigma\left(x_{m(n)}^{*}, F_{n}(\omega)\right)\right|+\left|\sigma\left(x_{m(n)}^{*}, F_{n}(\omega)\right)-\sigma\left(x^{*}, F(\omega)\right)\right| \\
& \quad \leq|| x^{*}-x_{m(n)}^{*} \||G(\omega)|+\left|\sigma\left(x_{m(n)}^{*}, F_{n}(\omega)\right)-\sigma\left(x^{*}, F(\omega)\right)\right|
\end{aligned}
$$

for all $\omega \in \Omega \backslash N^{\prime}, \mu\left(N^{\prime}\right)=0$. Here $G(\omega)={\overline{\bigcup_{n>1} F_{n}(\omega)}}^{\mathrm{w}} \in P_{w k}(X)$ for all $\omega \in \Omega \backslash N_{1}, \mu\left(N_{1}\right)=0$ and $N^{\prime}=N \cup N_{1}$ (cf. hypothesis (1)). So we get

$$
\begin{array}{rr}
\lim \sigma\left(x^{*}, F_{n}(\omega)\right)=\sigma\left(x^{*}, F(\omega)\right) & \text { for all } \omega \in \Omega \backslash N^{\prime}, \mu\left(N^{\prime}\right)=0 \\
\Rightarrow w-\varlimsup \lim F_{n}(\omega) \subseteq F(\omega) \mu \text {-a.e. } & \text { (see Proposition } 4.1 \text { of [33]). }
\end{array}
$$

Next note that

$$
\begin{aligned}
f_{n}^{k}(\omega) & =m_{n}^{k}(\omega)+p_{n}^{k}(\omega) \stackrel{s}{\rightarrow} m^{k}(\omega) \quad \mu \text {-a.e. } \\
& \Rightarrow m^{k}(\omega) \in s-\underline{\lim } F_{n}(\omega) \quad \mu \text {-a.e. }
\end{aligned}
$$

Since $s-\underline{\lim } F_{n}(\omega) \in P_{f c}(X)$, we have that

$$
F(\omega)=\overline{\operatorname{conv}}\left\{m^{k}(\omega)\right\}_{k \geq 1} \subseteq s-\underline{\lim } F_{n}(\omega) \quad \mu \text {-a.e. }
$$

From (4) and (5) above we conclude that $F_{n}(\omega) \stackrel{\mathrm{K}-\mathrm{M}}{\rightarrow} F(\omega) \mu$-a.e. Q.E.D.

From the proof of Theorem 14 , we have that $F_{n}(\omega) \stackrel{w}{\rightarrow} F(\omega) \mu$-a.e. In fact in the next theorem, we show that we can have this without the separability of $X^{*}$. 
Theorem 15. If $F_{n}: \Omega \rightarrow P_{f c}(X)$ are $\Sigma_{n}$-measurable multifunctions such that

(1) $\left\{F_{n}, \Sigma_{n}\right\}_{n \geq 1}$ is a set-valued uniform amart,

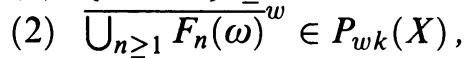

(3) $\left\{\left|F_{n}\right|\right\}_{n \geq 1}$ is uniformly integrable,

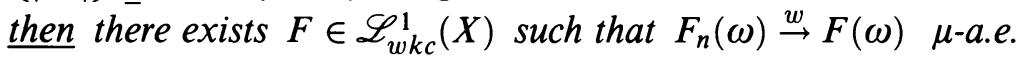

Proof. From Proposition 1.6 of Luu [25], we know that there exists a set-valued martingale $\left\{M_{n}, \Sigma_{n}\right\}_{n \geq 1}, M_{n} \in \mathscr{L}_{f c}^{1}(X)$ such that $\lim _{\tau \in T} \Delta\left(F_{\tau}, M_{\tau}\right)=0$. Also Theorem 3.2 of [34] (see also Theorem 3.2 of Wang-Xue [45]) tells us that there exists $F \in \mathscr{L}_{f c}^{1}(\Sigma, X)$ such that $M_{n}=E^{\Sigma_{n}} F$. Then for every $A \in \Sigma$ and for every $x^{*} \in X^{*}$, we have

$$
\int_{A}\left|\sigma\left(x^{*}, F_{n}(\omega)\right)-\sigma\left(x^{*}, E^{\Sigma_{n}} F(\omega)\right)\right| d \mu(\omega) \rightarrow 0 \quad \text { as } n \rightarrow \infty .
$$

But from Theorem 9 we know that

$$
\sigma\left(x^{*}, E^{\Sigma_{n}} F(\omega)\right)=E^{\Sigma_{n}} \sigma\left(x^{*}, F(\omega)\right)
$$

for all $\omega \in \Omega \backslash N, \mu(N)=0$ and all $x^{*} \in X^{*}$. So from Levy's theorem, we know that for every $x^{*} \in X^{*}, E^{\Sigma_{n}} \sigma\left(x^{*}, F(\cdot)\right) \rightarrow \sigma\left(x^{*}, F(\cdot)\right)$ in $L^{1}(\Omega)$. Hence for every $A \in \Sigma$ we have

$$
\begin{aligned}
& \int_{A}\left|\sigma\left(x^{*}, F_{n}(\omega)\right)-\sigma\left(x^{*}, F(\omega)\right)\right| d \mu(\omega) \\
& \leq \int_{A}\left|\sigma\left(x^{*}, F_{n}(\omega)\right)-E^{\Sigma_{n}} \sigma\left(x^{*}, F(\omega)\right)\right| d \mu(\omega) \\
&+\int_{A}\left|E^{\Sigma_{n}} \sigma\left(x^{*}, F(\omega)\right)-\sigma\left(x^{*}, F(\omega)\right)\right| d \mu(\omega) \rightarrow 0
\end{aligned}
$$

as $n \rightarrow \infty$. On the other hand, for every $x^{*} \in X^{*},\left\{\sigma\left(x^{*}, F_{n}(\cdot)\right), \Sigma_{n}\right\}_{n \geq 1}$ is a real-valued amart. So from Theorem 2.3 of Edgar-Sucheston [14], we have that if $D^{*}$ is a countable subset of $X^{*}$ which is dense in the Mackey topology $m\left(X^{*}, X\right)$ (it exists since $X$ is separable; cf. Wilansky [46, p. 144]), then for all $x^{*} \in D^{*}$ and all $\omega \in \Omega \backslash N, \mu(N)=0$ we have $\sigma\left(x^{*}, F_{n}(\omega)\right) \rightarrow u\left(\omega, x^{*}\right)$. So for all $A \in \Sigma$ and all $x^{*} \in D^{*}$

$$
\begin{aligned}
& \int_{A} \sigma\left(x^{*}, F(\omega)\right) d \mu(\omega)=\int_{A} u\left(\omega, x^{*}\right) d \mu(\omega) \\
& \quad \Rightarrow \sigma\left(x^{*}, F(\omega)\right)=u\left(\omega, x^{*}\right) \quad \text { for all }\left(\omega, x^{*}\right) \in\left(\Omega \backslash N_{1}\right) \times D^{*}, \mu\left(N_{1}\right)=0 .
\end{aligned}
$$

Because of hypothesis (2), $F(\omega) \in G(\omega) \omega \in \Omega \backslash N_{2}, \mu\left(N_{2}\right)=0$ with $G(\omega)=\overline{\operatorname{conv}}\left[\bigcup_{n \geq 1} F_{n}(\omega) \cup\left(-\bigcup_{n \geq 1} F_{n}(\omega)\right)\right] \in P_{w k c}(X)$. Now let $y^{*} \in X^{*}$. Then we can find a net $\left\{x_{\alpha}^{*}\right\}_{\alpha \in J}$ in $D^{*}$ such that $x_{\alpha}^{*} \rightarrow y^{*}$ in $m\left(X^{*}, X\right)$. For all $\omega \in \Omega \backslash N, N=N_{1} \cup N_{2}, \mu(N)=0$, we have

$$
\begin{aligned}
\mid \sigma\left(y^{*},\right. & \left.F_{n}(\omega)\right)-\sigma\left(y^{*}, F(\omega)\right) \mid \\
\leq & \left|\sigma\left(y^{*}, F_{n}(\omega)\right)-\sigma\left(x_{\alpha}^{*}, F_{n}(\omega)\right)\right|+\left|\sigma\left(x_{\alpha}^{*}, F_{n}(\omega)\right)-\sigma\left(x_{\alpha}^{*}, F(\omega)\right)\right| \\
& +\left|\sigma\left(x_{\alpha}^{*}, F(\omega)\right)-\sigma\left(y^{*}, F(\omega)\right)\right| \\
\leq & 2 \sigma\left(y^{*}-x_{\alpha}^{*}, G(\omega)\right)+\left|\sigma\left(x_{\alpha}^{*}, F_{n}(\omega)\right)-u\left(\omega, x_{\alpha}^{*}\right)\right| .
\end{aligned}
$$

Recall that $\left|\sigma\left(x_{\alpha}^{*}, F_{n}(\omega)\right)-u\left(\omega, x_{\alpha}^{*}\right)\right| \rightarrow 0$ as $n \rightarrow \infty$ for all $\alpha \in J$. Also $\sigma(\cdot, G(\omega))$ is $m$-continuous. Hence we deduce that for all $\omega \in \Omega \backslash N, \mu(N)=0$ 
and all $y^{*} \in X^{*}$

$$
\sigma\left(y^{*}, F_{n}(\omega)\right) \rightarrow \sigma\left(y^{*}, F(\omega)\right) \Rightarrow F_{n}(\omega) \stackrel{w}{\rightarrow} F(\omega) \quad \mu \text {-a.e. Q.E.D. }
$$

A careful reading of the above proof reveals that we can drop hypothesis (2) at the expense of reintroducing the separability hypothesis on the dual space $X^{*}$. So we have:

Theorem 16. If $X$ has the RNP, $X^{*}$ is separable and $F_{n}: \Omega \rightarrow P_{f c}(X)$ are $\Sigma_{n}$-measurable multifunctions such that

(1) $\left\{F_{n}, \Sigma_{n}\right\}_{n \geq 1}$ is a set-valued uniform amart,

(2) $\left\{\left|F_{n}\right|\right\}_{n \geq 1}$ is uniformly integrable,

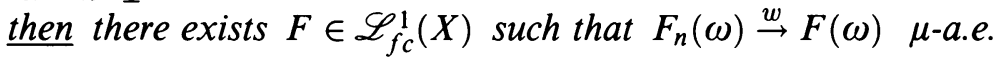

Let $\widehat{\mathscr{L}}_{f c}^{1}(X)$ be the closure of the set of simple multifunctions in the metric space $\left(\mathscr{L}_{f c}^{1}(X), \Delta\right)$. It is easy to see that $F \in \mathscr{L}_{f c}^{1}(X)$ if and only if, for $\mu$-almost all $\omega \in \Omega, F(\omega)$ belongs to a separable subspace of $\left(P_{f c}(X), h\right)$. If $\operatorname{dim} X<\infty$, from the Radstrom embedding theorem (see Klein-Thompson [22]), we have that $\widehat{\mathscr{L}}_{f c}^{1}(X)=\mathscr{L}_{f c}^{1}(X)$. Using an argument of Neveu [27], we can have the following improvement of Theorem 16 provided we know that the limit random set, guaranteed by that result, belongs in $\mathscr{L}_{f c}^{1}(X)$.

Theorem 17. If the hypotheses of Theorem 16 hold and $F \in \widehat{\mathscr{L}}_{f c}^{1}(X)$, then $F_{n}(\omega) \stackrel{h}{\rightarrow} F(\omega)$ M-a.e.

Proof. Let $C \in P_{f c}(X)$ and let $\left\{M_{n}, \Sigma_{n}\right\}_{n \geq 1}$ be the $P_{f c}(X)$-valued martingale as in the proof of Theorem 15. Let $D_{1}^{*}$ be a countable dense subset of the unit ball in $X^{*}$. Then applying Lemma 4 of Neveu [27] on the positive submartingale $\left\{\left|\sigma\left(x^{*}, M_{n}(\cdot)\right)-\sigma\left(x^{*}, C\right)\right|, \Sigma_{n}\right\}_{n \geq 1}, x^{*} \in D_{1}^{*}$, we get that for all $\omega \in \Omega \backslash N_{1}, \mu\left(N_{1}\right)=0$,

$$
\begin{aligned}
& h\left(M_{n}(\omega), C\right)=\sup _{x^{*} \in D_{i}^{*}}\left|\sigma\left(x^{*}, M_{n}(\omega)\right)-\sigma\left(x^{*}, C\right)\right| \\
& \rightarrow \sup _{x^{*} \in D_{i}^{*}}\left|\sigma\left(x^{*}, F(\omega)\right)-\sigma\left(x^{*}, C\right)\right|=h(F(\omega), C) .
\end{aligned}
$$

Since $F \in \widehat{\mathscr{L}}_{f c}^{1}(X)$ for all $\omega \in \Omega \backslash N_{2}, \mu\left(N_{2}\right)=0, F(\omega)$ belongs in a separable subset $\mathscr{D}$ of $\left(P_{f c}(X), h\right)$. So for every $A \in \mathscr{D}$ and all $\omega \in \Omega \backslash N$, $N=N_{1} \cup N_{2}, \mu\left(N_{2}\right)=0$ we have

$$
h\left(M_{n}(\omega), A\right) \rightarrow h(F(\omega), A) \quad \text { as } n \rightarrow \infty .
$$

Let $A=F(\omega)$. Then we have that $h\left(M_{n}(\omega), F(\omega)\right) \rightarrow 0 \quad \mu$-a.e. Recall that $\lim _{\tau \in T} \Delta\left(F_{\tau}, M_{\tau}\right)=0$. So invoking Theorem 2.3 of Edgar-Sucheston [14], we conclude that $h\left(F_{n}(\omega), F(\omega)\right) \rightarrow 0 \quad \mu$-a.e. Q.E.D.

Since if $\operatorname{dim} X<\infty$, we have $\widehat{\mathscr{L}}_{f c}^{1}(X)=\mathscr{L}_{f c}^{1}(X)$, we get the following interesting corollary.

Corollary 18. If $\operatorname{dim} X<\infty$ and $F_{n}: \Omega \rightarrow P_{f c}(X)$ are $\Sigma_{n}$-measurable multifunctions such that

(1) $\left\{F_{n}, \Sigma_{n}\right\}$ is a set-valued uniform amart, 
(2) $\left\{\left|F_{n}\right|\right\}_{n \geq 1}$ is uniformly integrable,

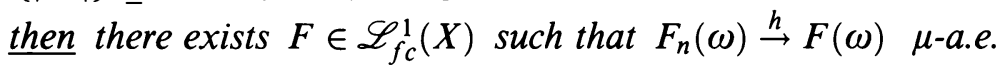

Remark. Theorem 17 extends to set-valued uniform amarts Theorem 3.3 of Wang-Xue [45]. Also it extends the earlier results of Neveu [27] and Daures [10].

Next we turn our attention to set-valued amarts and we prove two convergence results. The first of those is an alternative version of Theorem 5.1 in [34]. Namely we no longer assume that $X$ has the RNP and $X^{*}$ is separable, but our boundedness hypothesis is now stronger.

Theorem 19. If $F_{n}: \Omega \rightarrow P_{f c}(X)$ are $\Sigma_{n}$-measurable multifunctions such that

(1) $\left\{F_{n}, \Sigma_{n}\right\}_{n \geq 1}$ is a set-valued amart,

(2) $F_{n}(\omega) \subseteq G(\omega)$ u-a.e. with $G(\cdot) \in \mathscr{L}_{w k c}^{1}(X)$,

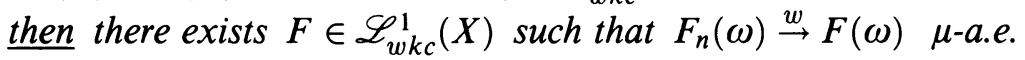

Proof. Let $D_{0}^{*} \subseteq X^{*}$ be a countable set which is dense in $X^{*}$ for the Mackey topology. Let $D^{*}=\operatorname{span}_{\mathbb{Q}} D_{0}^{*}=\{$ Rational linear combinations of elements in $\left.D_{0}^{*}\right\}$. Clearly this set is countable and dense in $X^{*}$ for the Mackey topology $m\left(X^{*}, X\right)$. Using Theorem 9 , we can easily check that, for every $x^{*} \in D^{*}$, $\left\{\sigma\left(x^{*}, F_{n}(\cdot)\right), \Sigma_{n}\right\}_{n \geq 1}$ is a real-valued amart. Then from Theorem 2.3 of EdgarSucheston [14] and since $D^{*}$ is countable, we have

$$
\sigma\left(x^{*}, F_{n}(\omega)\right) \rightarrow u\left(\omega, x^{*}\right)
$$

for all $\left(\omega, x^{*}\right) \in(\Omega \backslash N) \times D^{*}, \mu(N)=0$. Note that for every $x^{*}, y^{*} \in D^{*}$, we have

$$
\left|\sigma\left(x^{*}, F_{n}(\omega)\right)-\sigma\left(y^{*}, F_{n}(\omega)\right)\right| \leq\left|\sigma\left(x^{*}-y^{*}, F_{n}(\omega)\right)\right| \leq \sigma\left(x^{*}-y^{*}, \widehat{G}(\omega)\right)
$$

where $\widehat{G}(\omega)=\overline{\operatorname{conv}}[G(\omega) \cup(-G(\omega))], \widehat{G}(\cdot) \in \mathscr{L}_{w k c}^{1}(X)$ and has symmetric values. Passing to the limit as $n \rightarrow \infty$, we get

$$
\left|u\left(\omega, x^{*}\right)-u\left(\omega, y^{*}\right)\right| \leq \sigma\left(x^{*}-y^{*}, \widehat{G}(\omega)\right), \quad x^{*}, y^{*} \in D^{*} .
$$

Recalling that $\sigma(\cdot, \widehat{G}(\omega))$ is $m$-continuous (cf. Laurent [24]), we deduce from the above inequality that, for all $\omega \in \Omega \backslash N, \mu(N)=0, u(\omega, \cdot)$ is $m\left(X^{*}, X\right)$-continuous on $D^{*}$. Furthermore from Theorem 5.3, page 216 of Dugundji [12], we deduce that for all $\omega \in \Omega \backslash N, \mu(N)=0, u(\omega, \cdot)$ has a unique $m\left(X^{*}, X\right)$-extension $\hat{u}\left(\omega, x^{*}\right)$ on all of $X^{*}$. It is easy to see that $\hat{u}(\omega, \cdot)$ is sublinear. So there exists $F(\omega) \in P_{w k c}(X)$ such that $\hat{u}\left(w, x^{*}\right)=$ $\sigma\left(x^{*}, F(\omega)\right)$. We claim that for all $\left(\omega, x^{*}\right) \in(\Omega \backslash N) \times X^{*}, \mu(N)=0$ we have $\sigma\left(x^{*}, F_{n}(\omega)\right) \rightarrow \sigma\left(x^{*}, F(\omega)\right)$. To see this, let $\left\{z_{\beta}^{*}\right\}_{\beta \in \Gamma} \subseteq D^{*}$ such that $z_{\beta}^{*} \stackrel{m}{\rightarrow} x^{*}$. We have

$$
\begin{aligned}
\mid \sigma\left(x^{*},\right. & \left.F_{n}(\omega)\right)-\sigma\left(x^{*}, F(\omega)\right)|\leq| \sigma\left(x^{*}, F_{n}(\omega)\right)-\sigma\left(z_{\beta}^{*}, F_{n}(\omega)\right) \mid \\
& +\left|\sigma\left(z_{\beta}^{*}, F_{n}(\omega)\right)-\sigma\left(z_{\beta}^{*}, F(\omega)\right)\right|+\left|\sigma\left(z_{\beta}^{*}, F(\omega)\right)-\sigma\left(x^{*}, F(\omega)\right)\right| \\
\leq & 2 \sigma\left(x^{*}-z_{\beta}^{*}, \widehat{G}(\omega)\right)+\left|\sigma\left(z_{\beta}^{*}, F_{n}(\omega)\right)-\sigma\left(z_{\beta}^{*}, F(\omega)\right)\right| \\
& +\left|\sigma\left(z_{\beta}^{*}-x^{*}, F(\omega)\right)\right| .
\end{aligned}
$$

Since $\sigma(\cdot, \widehat{G}(\omega))$ is $m$-continuous and $\sigma\left(z^{*}, F_{n}(\omega)\right) \rightarrow \sigma\left(z^{*}, F(\omega)\right)$ for all $\left(\omega, z^{*}\right) \in(\Omega \backslash N) \times D^{*}, \mu(N)=0$, we get that $\sigma\left(x^{*}, F_{n}(\omega)\right) \rightarrow \sigma\left(x^{*}, F(\omega)\right)$ 
for all $\left(\omega, x^{*}\right) \in(\Omega \backslash N) \times X, \mu(N)=0$. Therefore $F_{n}(\omega) \stackrel{w}{\rightarrow} F(\omega) \mu$ a.e. Q.E.D.

The final convergence result deals with $P_{k c}(X)$-valued amarts.

Theorem 20. If $F_{n}: \Omega \rightarrow P_{k c}(X)$ are $\Sigma_{n}$-measurable multifunctions such that

(1) $\left\{F_{n}, \Sigma_{n}\right\}_{n \geq 1}$ is a set-valued amart,

(2) $F_{n}(\omega) \subseteq G(\omega)$ u-a.e. with $G \in \mathscr{L}_{k c}^{1}(X)$,

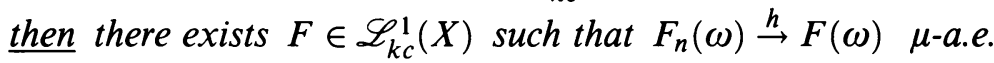

Proof. Using the notation of the proof of Theorem 20, we have

$$
\left|\sigma\left(x^{*}, F_{n}(\omega)\right)-\sigma\left(y^{*}, F_{n}(\omega)\right)\right| \leq \sigma\left(x^{*}-y^{*}, \widehat{G}(\omega)\right)
$$

and from the Banach-Dieudonné theorem we know that $\sigma(\cdot, \widehat{G}(\omega))$ is continuous from $B_{w^{*}}^{*}$ into $\mathbb{R}$, where $B_{w^{*}}^{*}$ denotes the unit ball of $X^{*}$ equipped with the $w^{*}$-topology (note that, by Mazur's theorem, $\widehat{G}(\cdot) \in \mathscr{L}_{k c}^{1}(X)$ ). So for every $\omega \in \Omega \backslash N, \mu(N)=0,\left\{\sigma\left(\cdot, F_{n}(\omega)\right)\right\}_{n \geq 1}$ is equicontinuous in $C\left(B_{w^{*}}^{*}\right)$ and by Theorem 19, $\sigma\left(x^{*}, F_{n}(\omega)\right) \rightarrow \sigma\left(x^{*}, F(\omega)\right)$ for all $\left(\omega, x^{*}\right) \in(\Omega \backslash N) \times X^{*}$, $\mu(N)=0$. So from the Arzela-Ascoli theorem, we deduce that $\sigma(\cdot, F(\omega)) \in$ $C\left(B_{w^{*}}^{*}\right)$ for all $\omega \in(\Omega \backslash N), \mu(N)=0$. Hence $F(\cdot) \in \mathscr{L}_{k c}^{1}(X)$ and

$$
\sup _{\left\|x^{*}\right\| \leq 1}\left|\sigma\left(x^{*}, F_{n}(\omega)\right)-\sigma\left(x^{*}, F(\omega)\right)\right| \rightarrow 0 \text { as } n \rightarrow \infty
$$

for all $\omega \in \Omega \backslash N, \mu(N)=0$. Thus by Hörmander's formula, we get $F_{n}(\omega) \stackrel{h}{\rightarrow}$ $F(\omega) \quad \mu$-a.e. Q.E.D.

\section{ACKNOWLEDGMENTS}

The author wishes to thank a very knowledgeable referee for his (her) corrections and remarks on the original version. Also the author wishes to thank Professor R. Durrett for his kind and helpful attitude during the review process.

\section{REFERENCES}

1. R. Alo, A. de Korvin, and R. Roberts, The optional sampling theorem for convex set-valued martingales, J. Reine Angew. Math. 310 (1979), 1-6.

2. R. Ash, Real analysis and probability, Academic Press, New York, 1972.

3. H. Attouch, Variational convergence for functions and operators, Pitman, London, 1984.

4. D. Austin, G. Edgar, and A. Ionescu-Tulcea, Pointwise convergence in terms of expectations, Z. Wahrsch. Verw. Gebiete 30 (1974), 17-26.

5. S. Bagchi, On a.s. convergence of classes of multivalued asymptotic martingales, Ann. Inst. H. Poincaré 21 (1985), 313-321.

6. A. Bellow, Uniform amarts: a class of asymptotic martingales for which strong almost sure convergence obtains, Z. Wahrsch. Verw. Gebiete 41 (1978), 177-191.

7. J. Bourgain, An averaging result for $l^{1}$-sequences and applications to weakly conditionally compact sets in $L_{X}^{1}$, Israel J. Math. 32 (1979), 289-298.

8. A. Costé, Sur les martingales multivoques, C. R. Acad. Sci. Paris 290 (1980), 953-956.

9. B. K. Dam, Almost sure convergence of set-valued martingales and submartingales, Acta Math. Hungar. 60 (1992), 197-205.

10. J.-P. Daures, Version multivoque du théorème de Doob, Ann. Inst. H. Poincaré 9 (1973), $167-176$. 
11. J. Diestel and J. Uhl, Vector measures, Math. Surveys, vol. 15, Amer. Math. Soc., Providence, RI, 1977.

12. J. Dugundji, Topology, Allyn and Bacon, Boston, 1966.

13. E. Dynkin and I. Evstigneev, Regular conditional expectations of correspondences, Theor. Probab. Appl. 25 (1976), 325-338.

14. G. Edgar and L. Sucheston, Amarts: a class of asymptotic martingales. A: Discrete parameter, J. Multivariate Anal. 6 (1976), 193-221.

15. L. Egghe, Stopping time techniques for analysts and probabilists, Cambridge Univ. Press, Cambridge, UK, 1984.

16. A. Hanen and J. Neveu, Atomes conditionels d'un espace de probabilité, Acta Math. Hungar. 17 (1966), 443-449.

17. F. Hiai and H. Umegaki, Integrals, conditional expectations and martingales of multivalued functions, J. Multivariate Anal. 7 (1977), 149-182.

18. F. Hiai, Convergence of conditional expectations of strong laws of large numbers for multivalued random variables, Trans. Amer. Math. Soc. 291 (1985), 613-627.

19. L. Hörmander, Sur la fonction d'appui des ensembles convexes dans un espace localement convexe, Ark. Mat. 3 (1954), 181-186.

20. A. Ionescu-Tulcea and C. Ionescu-Tulcea, Topics in the theory of lifting, Springer, Berlin, 1969.

21. H.-A. Klei, A compactness criterion in $L^{1}(X)$ and Radon-Nikodym theorems for multimeasures, Bull. Sci. Math. 112 (1988), 305-324.

22. E. Klein and A. Thompson, Theory of correspondences, Wiley, New York, 1984.

23. A. de Korvin and R. Kleyle, A convergence theorem for convex set-valued supermartingales, Stochastic Anal. Appl. 3 (1985), 433-445.

24. P.-J. Laurent, Approximation et optimisation, Hermann, Paris, 1972.

25. D. Q. Luu, Quelques resultats de representation des amarts uniformes multivoques, C. R. Acad. Sci. Paris 300 (1985), 63-65.

26. K. Musial, The weak Radon-Nikodym property in Banach spaces, Studia Math. 64 (1979), 151-173.

27. J. Neveu, Convergence presque sûre des martingales multivoques, Ann. Inst. H. Poincaré 8 (1972), 1-7.

28. _ Discrete parameter martingales, North-Holland, Amsterdam, 1975.

29. N. S. Papageorgiou, On the theory of Banach space valued multifunctions. 1: Integration and conditional expectation, J. Multivariate Anal. 17 (1985), 185-206.

30. (1985), 115-136.

31. 452-479.

32. $\ldots$, A convergence theorem for set-valued submartingales with values in a separable Banach space, Stochastic Anal. Appl. 5 (1987), 510-520.

33. Convergence theorems for Banach space-valued integrable multifunctions, Internat $\mathrm{J}$. Math. Math. Sci. 10 (1987), 433-442.

34. Convergence and representation theorems for set-valued random processes, J. Math. Anal. Appl. 150 (1990), 129-145.

35. Convergence theorems for set-valued conditional expectations, Comment. Math. Univ. Carolin. 34 (1993), 97-104.

36. G. Pisier, Une propriété de stabilité de la classe des espaces ne contenant pas $l^{1}, \mathrm{C}$. R. Acad. Sci. Paris 286 (1978), 747-749.

37. H. Robbins, On the measure of a random set, Ann. Math. Statist. 15 (1944), 70-74.

38. __ On the measure of a random set, Ann. Math. Statist. 16 (1945), 342-347.

39. H. Rosenthal, A characterization of Banach spaces containing $l^{1}$, Proc. Nat. Acad. Sci. U.S.A. 71 (1974), 2411-2413. 
40. G. Salinetti and R. Wets, On the convergence of closed valued measurable multifunctions, Trans. Amer. Math. Soc. 266 (1981), 275-289.

41. $\ldots$, On the convergence in distribution of measurable multifunctions (random sets), normal integrands, stochastic processes and stochastic infima, Math. Oper. Res. 11 (1986), 385-419.

42. H. Schaefer, Topological vector spaces, Springer, New York, 1971.

43. M. Talagrand, Weak Cauchy sequences in $L^{1}(E)$, Amer. J. Math. 106 (1984), 703-724.

44. D. Wagner, Survey of measurable selection theorems, SIAM J. Control Optim. 15 (1977), 859-903.

45. Z.-P. Wang and X.-H. Xue, On convergence and closedness of multivalued martingales, Trans. Amer. Math. Soc. 341 (1994), 807-827.

46. A. Wilansky, Modern methods in topological vector spaces, McGraw-Hill, New York, 1978.

47. M. C. Yovitz, C. Foulk, and L. Rose, Information flow and analysis: Theory, simulation and experiment. Part I: Basic theoretical and conceptual development, Amer. Soc. Inf. Sci. 32 (1981), 187-202.

Department of Mathematics, National Technical University, Zografou Campus, Athens 15780, Greece (Permanent address)

Department of Applied Mathematics, Florida Institute of Technology, 150 West University Blvd., Melbourne, Florida 32901-6988 